DIW BERLIN

Discussion

Papers

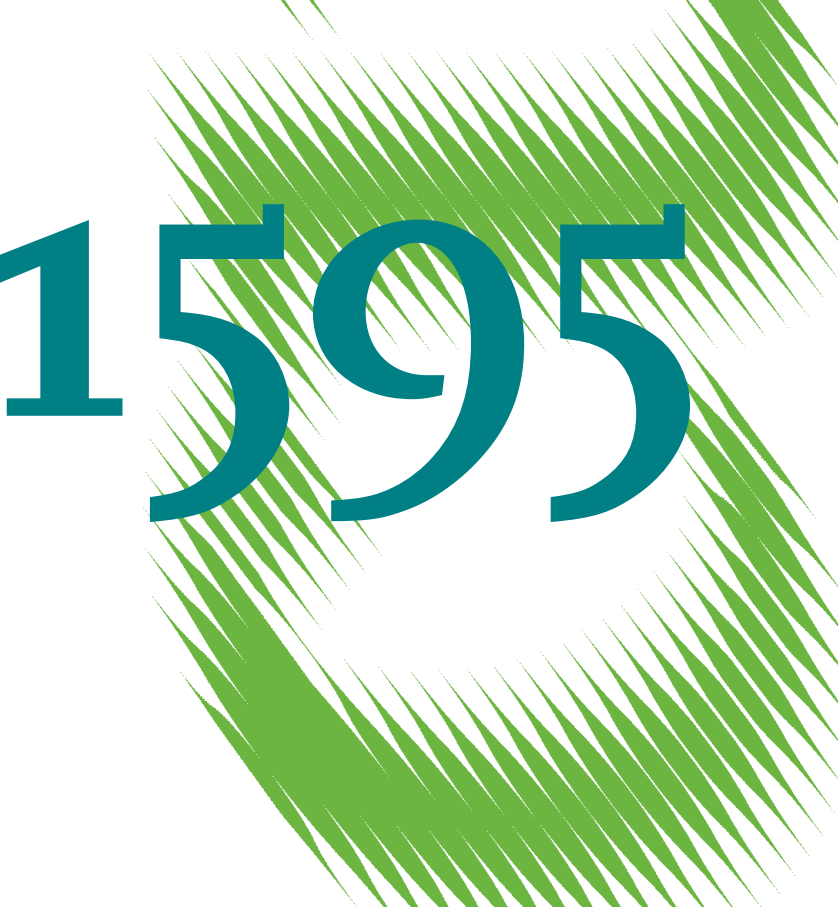

Herausforderungen bei der Messung von Wohlfahrt 
Opinions expressed in this paper are those of the author(s) and do not necessarily reflect views of the institute.

IMPRESSUM

(C) DIW Berlin, 2016

DIW Berlin

German Institute for Economic Research

Mohrenstr. 58

10117 Berlin

Tel. +49 (30) $89789-0$

Fax +49 (30) $89789-200$

http://www.diw.de

ISSN electronic edition 1619-4535

Papers can be downloaded free of charge from the DIW Berlin website:

http://www.diw.de/discussionpapers

Discussion Papers of DIW Berlin are indexed in RePEc and SSRN:

http://ideas.repec.org/s/diw/diwwpp.html

http://www.ssrn.com/link/DIW-Berlin-German-Inst-Econ-Res.html 


\title{
Herausforderungen bei der Messung von Wohlfahrt
}

\author{
Beate Jochimsen* und Christian Raffer
}

Zusammenfassung: Initiiert von einer lebhaften öffentlichen und wissenschaftlichen Debatte um die Eignung des Bruttoinlandsprodukts als Indikator für materiellen Wohlstand sind zahlreiche alternative Maße zur Wohlstands- und Wohlfahrtsmessung entwickelt worden. Fällt dabei die Wahl auf einen Indikatorensatz, ist die Bestimmung der optimalen Anzahl der Indikatoren eine zentrale Herausforderung. Je mehr Indikatoren ausgewählt werden, desto besser können zwar die Bestandteile der Wohlfahrt abgebildet werden, desto schlechter ist aber die Kommunizierbarkeit. Beate Jochimsen und Christian Raffer analysieren mit Hilfe mehrerer statistischer Methoden die Verbindungen der $10 \mathrm{~W}^{3}$-Leitindikatoren des Deutschen Bundestages untereinander. Es zeigt sich, dass - je nach Messverfahren - drei bis sechs dieser Indikatoren ohne großen Informationsverlust weggelassen werden können, da sie mit den verbleibenden eng korreliert sind. Ein kleiner $W^{3}$-Indikatorensatz könnte wesentlich leichter erklärt und kommuniziert werden. Im Ergebnis bedeutet dies, dass der Umfang aller nationaler und internationaler Indikatorensätze zur Wohlfahrtsmessung kritisch zu würdigen ist.

JEL-Klassifikation: I31, D6, E01, C18, I32, Y1

Schlüsselwörter: Wohlfahrtsmessung, Bruttoinlandsprodukt, W3-Indikatorensatz, Korrelations- und Hauptkomponentenanalyse

*Kontaktperson: Beate Jochimsen, Professur für Volkswirtschaftslehre, insb. Finanzwissenschaft, Hochschule für Wirtschaft und Recht Berlin, Badensche Str. 52, 10825 Berlin, sowie Forschungsdirektorin für Föderalismusanalyse, Deutsches Institut für Wirtschaftsforschung e.V., E-Mail: beate.jochimsen@hwr-berlin.de

Christian Raffer, Humboldt Universität Berlin sowie Hochschule für Wirtschaft und Recht Berlin, privat: Karl-Marx-Allee 131, 10243 Berlin, E-Mail: rafferch@hu-berlin.de 


\section{Challenges of Welfare Measurement}

Abstract: Triggered by the lively public and academic debate on the gross domestic product as welfare indicator numerous alternative welfare measurements emerged. If a set of indicators as welfare measurement is chosen the identification of an optimal number of indicators is a challenging question. The more indicators are included in the set the more comprehensive welfare can be mapped, thus, the more complicated is the communication of results. Based on several statistical methods Beate Jochimsen and Christian Raffer analyze the linkage of the ten $\mathrm{W}^{3}$-Indicators which the German Bundestag developed. They find that - depending on the statistical method - between three and six of those ten indicators could be easily excluded as they are closely correlated with the remaining ones. A smaller set of $W^{3}$-Indicators could be explained far easier to the public. This result suggests to critically evaluate the number of indicators in various welfare measures on national and international level.

Keywords: Welfare measurement, gross domestic product, $\mathrm{W}^{3}$-Indicators, correlation and principle component analysis 


\section{Wohlstandsmessung jenseits des BIPs}

Bereits seit Längerem gibt es eine lebhafte öffentliche, politische und wissenschaftliche Debatte um die Eignung des Bruttoinlandsprodukts (BIP) als Indikator für materiellen Wohlstand und - mehr noch - gesamtgesellschaftliche Wohlfahrt $^{1}$ (Schmidt/Aus dem Moore 2012: 58). Obwohl das BIP als Wohlfahrtsmaß nie gedacht war, ist es unter Tolerierung der Wissenschaft und Statistik von Politik und Öffentlichkeit dazu gemacht worden (Enquete 2013: 233f., Diefenbacher 2013: 66f.). Kritisch diskutiert werden vor allem folgende Aspekte: Als Bruttomaß enthält das BIP Abschreibungen des Kapitalstocks und lässt von Inländern im Ausland erzielte sowie von Ausländern übertragene Einkommen unbeachtet. Es erfasst Qualitätsveränderungen, bereitgestellte öffentliche Güter und Dienstleistungen sowie nicht marktvermittelte Produktion nur ungenau. Schäden wirtschaftlicher Aktivität, die die Wohlfahrt mindern, werden unangemessen, nicht-materielle Gegebenheiten wie Gesundheit überhaupt nicht eingerechnet. Hinzu kommt, dass Verteilungsaspekte vom BIP nicht abgebildet werden.

Parallel zu der immer lauter werdenden Debatte um die Sinnhaftigkeit einer zu starken Fixierung auf das Wirtschaftswachstum entstand im Jahr 1972 mit dem „Measure of Economic Welfare" der erste alternative Ansatz der Wohlfahrtsmessung, konzipiert von William Nordhaus und James Tobin (Schmidt/Aus dem Moore 2012: 59). Aus der Motivation heraus, die gesellschaftliche Wohlfahrt über ein breiteres statistisches Messkonzept besser fassen zu können, ist bis heute sowohl auf internationaler wie auch auf nationaler und sogar kommunaler Ebene eine gleichermaßen große wie unübersichtliche Anzahl alternativer Maße entstanden (Kaether et al. 2005: 27-34, Enquete 2013: 302-330). Zu besonderer Bekanntheit brachten es unter anderem der Human Development Index (HDI) der Vereinten Nationen und - auf deutscher Ebene - der Nationale Wohlfahrtsindex (NWI) von Hans Diefenbacher und Roland Zieschank. Neuen Schwung erhielt das Thema im Jahr 2009, als eine Expertenkommission um die Ökonomen Joseph Stiglitz, Amartya Sen und Jean-Paul Fitoussi im Auftrag der französischen Regierung einen Bericht mit umfassenden Empfehlungen erweiterter Wohlfahrtsmessung publizierte und 2010

\footnotetext{
${ }^{1}$ In der vorliegenden Arbeit werden die Begriffe „Wohlfahrt“ und „Wohlstand“ in klarer Abgrenzung voneinander verwendet. In Anlehnung an Bardt et al. (2012: 48) meint die Wohlfahrt neben dem rein materiellen Wohlstand auch die Bedeutung immaterieller Gegebenheiten. Wohlstand ist damit die materielle Dimension der Wohlfahrt; mithin die Möglichkeit, über Waren und Dienstleistungen zu verfügen.
} 
ein Gutachten der deutschen und französischen Sachverständigenräte folgte (Stiglitz et al. 2009, SVR 2010, Bardt et al. 2012: 48, Schmidt/Aus dem Moore 2012: 62). Vor diesem Hintergrund setzte der Deutsche Bundestag im Jahr 2010 die EnqueteKommission „Wachstum, Wohlstand, Lebensqualität - Wege zu nachhaltigem Wirtschaften und gesellschaftlichem Fortschritt in der Sozialen Marktwirtschaft" ein.

Allen Initiativen gemein ist, dass sie zu Beginn ihrer Arbeit die grundlegende Frage beantworten müssen, ob Wohlstand in einem Einheitsindikator ${ }^{2}$ (wie beispielsweise dem HDI) oder in einem Indikatorensatz (z.B. der Nachhaltigkeitsstrategie der Bundesregierung (Nachhaltigkeitsrat 2010)) gemessen werden soll. Wenn die Entscheidung für einen Indikatorensatz gefallen ist, muss entschieden werden, welche Indikatoren und vor allem wie viele Indikatoren zum Satz gehören sollen. Dabei steht man einem Trade-off zwischen öffentlicher Wahrnehmung der neuen Messgrößen und Abbildung der Komplexität des Lebens gegenüber. Ersteres spricht für wenige Indikatoren, die in Summe intensiver und eher öffentlich wahrgenommen werden, letzteres für viele Indikatoren, die die Individualität der Menschen widerspiegeln.

Dieses Spannungsverhältnis kann gemildert werden, indem untersucht wird, inwiefern die einzelnen Indikatoren miteinander korreliert sind. Bei enger Korrelation von zwei Indikatoren mag die Einfügung beider zwar einen psychologischen Mehrwert dahingehend bringen, dass zwei Aspekte der Wohlfahrt erfasst werden. Inhaltlich wäre jedoch kein Zugewinn zu verzeichnen, da die Entwicklung des einen Indikators infolge einer engen Korrelation mit dem zweiten Indikator dessen Entwicklung mit abbildet.

Die Enquête-Kommission „Wachstum, Wohlstand, Lebensqualität" hat einen Indikatorensatz zur Wohlfahrtssmessung entwickelt, die W'3-Indikatoren. Inwiefern die Auswahl dieser Indikatoren den Ansprüchen an die - vor dem Hintergrund ökonomischer oder statistischer Kriterien - optimale Zahl von Indikatoren entspricht, wird in dieser Arbeit untersucht. Dafür werden die W33-Leitindikatoren auf Grundlage einer Korrelations- und einer Hauptkomponentenanalyse empirisch auf Reduktionsmöglichkeiten hin geprüft. Betrachtet wird Deutschland für die Jahre 1990 bis 2012 sowie alle Länder der EU-28 für das Jahr 2008. Die Ergebnisse zeigen,

\footnotetext{
${ }^{2}$ Eine vertiefte Systematik hinsichtlich der weitergehenden Unterteilung von hochaggregierten Indizes, i.e. ,composite indicators' (in BIP-Revisionen, Subjektive/Objektive Indizes, usw.) findet sich in: Van Suntum/Lerbs 2011: 41.
} 
dass angesichts der Beziehungen einzelner Indikatoren untereinander Spielraum für eine Reduktion der $\mathrm{W}^{3}$-Leitindikatoren besteht, ohne damit einen zu großen Informationsverlust zu erleiden.

\section{Die Enquête-Kommission und die $\mathbf{W}^{3}$-Indikatoren}

\subsection{Auftrag und Indikatorensatz}

Auftrag der Enquete-Kommission „Wachstum, Wohlstand, Lebensqualität - Wege zu nachhaltigem Wirtschaften und gesellschaftlichem Fortschritt in der Sozialen Marktwirtschaft“ war es unter anderem, einen „alternativen Wohlstandsindikator“ zu entwickeln (Enquete 2013: 803ff.). Mit den sogenannten $\mathrm{W}^{3}$-Indikatoren hat die Kommission - im Besonderen deren Projektgruppe 2 - im Jahr 2013 einen Indikatorensatz vorgelegt, der ein umfassendes Bild gesamtgesellschaftlicher Wohlfahrt vermitteln soll (Enquete 2013: 276). Bei den $W^{3}$-Indikatoren handelt es sich um einen Indikatorensatz, nicht einen hochaggregierten Index (Enquete 2013: 236). Die Enquete-Kommission hat sich explizit dagegen entschieden, einen Index zu bilden, in dem verschiedene Einzelindikatoren statistisch genormt, gewichtet und verrechnet werden, um am Ende eine leicht zu kommunizierende Zahl zu erhalten (Enquete 2013: 234). Um mit einem Indikatorensatz ein möglichst verständliches Bild gesamtgesellschaftlichen Wohlergehens vermitteln zu können, ist es wichtig, dass mit der Hinzunahme weiterer Indikatoren ein Erkenntnisgewinn einhergeht (SVR 2010: 69). Anders ausgedrückt sollte die statistische Korrelation unter den einzelnen Indikatoren nicht zu eng sein, um das Set vor einer überflüssigen Aufblähung zu bewahren. Diesem Punkt kommt eine zentrale Bedeutung zu, da ein Indikatorensatz immer im Konflikt steht, so wenige Indikatoren wie möglich und so viele wie nötig einzubeziehen, um einerseits die Ergebnisse einer breiten Öffentlichkeit vermitteln und andererseits das komplexe Phänomen gesellschaftlicher Wohlfahrt hinreichend abbilden zu können (Grunwald/Kopfmüller 2012: 84).

Die $\mathrm{W}^{3}$-Indikatoren gliedern sich in die drei Wohlfahrts-Dimensionen "Materieller Wohlstand", „Soziales und Teilhabe" sowie "Ökologie“. Diese Dimensionen - die in der Nachhaltigkeitsdiskussion als die drei klassischen Dimensionen der Nachhaltigkeit gelten (Diefenbacher 2009: 683f.) - wurden deduktiv mit überwiegend objektiven Einzelindikatoren bestückt; allein der Indikator „Voice \& Accountability" stützt sich auf subjektive Daten. Die Kommission unterscheidet Leitindikatoren, Warnlampen und Hinweislampen (Enquete 2013: 237). Während die 
Leitindikatoren die zentralen Bestandteile des Sets sind und in jeder Berichtsperiode veröffentlicht werden sollen, werden die „Warnlampen-Indikatoren“ lediglich im Hintergrund beobachtet und nur im Bedarfsfall kommuniziert. Einem Indikator kommt der Status der „Hinweislampe“ zu. Aufgrund schlechter Datenverfügbarkeit soll dieser Indikator immer dann veröffentlicht werden, wenn neue Daten vorliegen.

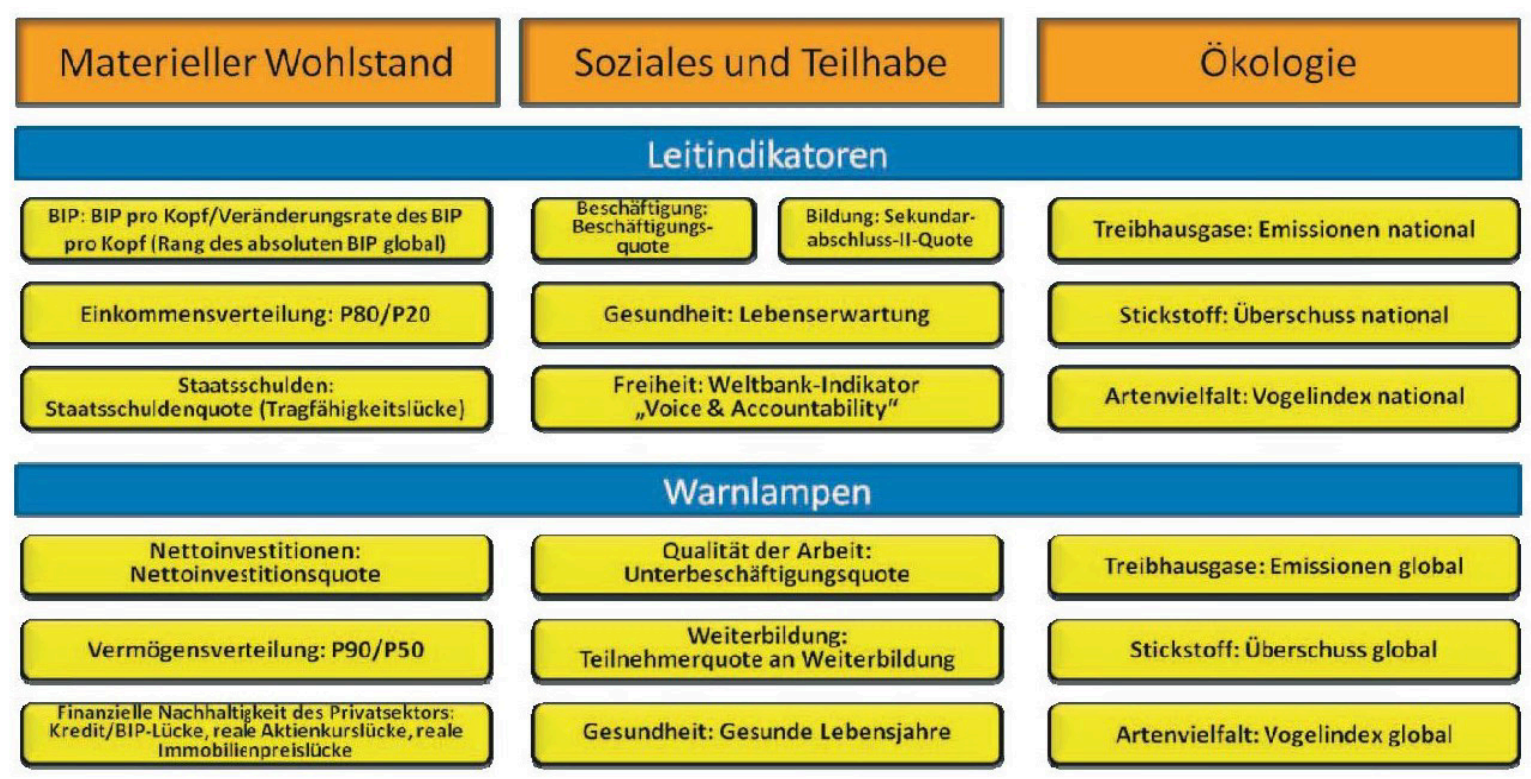

Hinweislampe

Nicht-marktvermittelte Produktion

\section{Abbildung 1: Die $W^{3}$-Indikatoren im Detail}

Quelle: Enquete 2013: 276.

Im Folgenden werden aus Gründen des Umfangs ausschließlich die $\mathrm{W}^{\circ}$ Leitindikatoren untersucht, die nun detaillierter vorgestellt werden ${ }^{3}$.

\subsection{Die $\mathbf{W}^{3}$-Leitindikatoren}

Bruttoinlandsprodukt (BIP) pro Kopf/Veränderungsrate BIP pro Kopf: Aus Gründen der besseren internationalen Vergleichbarkeit hat sich die Enquete-Kommission dazu entschieden, primär den Pro-Kopf-Wert des Bruttoinlandsprodukts heranzuziehen. Als Maß für die gesamte wirtschaftliche Leistung einer Volkswirtschaft spiegelt er einen wesentlichen Teil des materiellen Wohlstands wider. Um sowohl internationale Niveauvergleiche im Zeitablauf anstellen als auch die nationale Entwicklung im Blick behalten zu können, werden neben den absoluten Werten die Veränderungsraten

\footnotetext{
${ }^{3}$ Für eine umfassende Erläuterung der jeweiligen Warnlampen- und Hinweislampen-Indikatoren: siehe Enquete 2013: 238-274.
} 
des BIP betrachtet. Ergänzend wird das gesamte reale BIP in einem internationalen Ranking angesehen.

Einkommensverteilung: Die Einkommensverteilung wird mit der PerzentilBetrachtung des Nettoäquivalenzeinkommens gemessen, konkret mit der Relation des 80. und des 20. Perzentils. Als Begründung für die Auswahl dieses Leitindikators und dieser Perzentile wurden seine intuitive Verständlichkeit sowie der relativ große Abstand der Perzentile zu den jeweiligen Extremwerten darüber und darunter angegeben. Dieser Indikator vermittelt, wie weit die Einkommen der „Bessergestellten“ und die der „Einkommensschwachen“ auseinander liegen.

Staatsschuldenquote: Der Leitindikator stellt die Quote der Brutto-Staatsschulden im Verhältnis zum BIP dar. Er ist einerseits international etabliert, andererseits kompatibel zu den Kriterien des Stabilitäts- und Wachstumspaktes der Europäischen Union. Den Aspekt der Nachhaltigkeit der Staatsverschuldung berücksichtigt die Kommission, indem sie die Fiskalische Nachhaltigkeitslücke als Ergänzung aufnimmt. Letztere beschreibt den potentiellen Gesamt-Konsolidierungsbedarf (heute und in der Zukunft) des Staatshaushaltes in Prozent des BIPs.

Beschäftigungsquote: Im Gegensatz zur national bekannteren Arbeitslosenquote ist die Beschäftigungsquote schwerer durch nationale Gesetzgebung oder arbeitsmarktpolitische Programme zu beeinflussen. Sie ist zudem international besser vergleichbar. Die Beschäftigungsquote gibt den prozentualen Anteil der Erwerbstätigen an der Bevölkerung im Alter von 15 bis 64 Jahren an. Erwerbstätig ist, wer im betrachteten Zeitraum mindestens eine Stunde gegen Lohn gearbeitet hat.

Abschlussquote im Sekundarbereich II: Um den Bildungsstand in der Bevölkerung zu erfassen, zieht die Enquete-Kommission die Quote aller 20- bis 24-Jährigen heran, die mindestens einen Abschluss im Sekundarbereich II haben (berufsqualifizierender Abschluss, Fach-, fachgebundene oder allgemeine Hochschulreife). Ein solcher Abschluss wird als „Mindestqualifikation für die sich fortentwickelnde Wissensgesellschaft angesehen“ (Enquete 2013: 260). Dem Leitindikator kommt damit eine Frühwarnfunktion zu.

Lebenserwartung bei Geburt: Der Leitindikator, der anhand nationaler Sterbetafeln errechnet wird, stellt die durchschnittliche Lebenserwartung bei Geburt dar. Andere 
Indikatoren dieses Bereiches - etwa die Anzahl der verlorenen potenziellen Lebensjahre - sind sehr komplex konstruiert und in weit entwickelten Gesellschaften in ihrer Varianz zudem schwach.

Voice \& Accountability: Der Weltbank-Indikator „Voice \& Accountability ist ein Index aus zahlreichen Einzelwerten. Er erfasst, „wie Bürgerinnen und Bürger eines Landes ihre Teilhabe an der Auswahl ihrer Regierung wahrnehmen, ebenso wird das vorherrschende Maß an Meinungs-, Koalitions- und Pressefreiheit erfasst" (Enquete 2013: 262). Bei den Einzelwerten handelt es sich ausschließlich um sekundär ausgewertete Umfrageergebnisse - also subjektive Daten - die von Experten, Bürgern und Unternehmen stammen. Diese Daten werden aggregiert und auf eine Skala von 0 bis 100 normiert; Dabei bezeichnet der Wert 100 den höchsten Grad an „Demokratie“.

Treibhausgasemissionen (national): Der Umweltleitindikator orientiert sich an den Vorgaben des Kyoto-Protokolls und verrechnet fünf unterschiedliche $\mathrm{Stoffe}^{4}$ in Kohlendioxid-Äquivalente, um sie anschließend zu den $\mathrm{CO}_{2}$-Emissionen zu addieren. Das Ergebnis ist die Treibhausgasemission in Tonnen pro Jahr.

Stickstoffüberschuss: Der Leitindikator bildet die Stickstoffzufuhr abzüglich der Stickstoffabfuhr pro Quadratmeter landwirtschaftlich genutzter Fläche ab und stellt einen Teilausschnitt der Umweltbelastung durch menschliche Produktion dar.

Vogelindex: Der Leitindikator bildet den Verlust an Biodiversität in Deutschland ab. Da es sich bei Artenvielfalt um ein komplexes Phänomen handelt, stellt der Vogelindex nur eine Annährung dar. Um die Werte international vergleichen zu können, hat sich die Enquete-Kommission dafür entschieden, einen Teilindex des Vogelindex in den $W^{3}$-Indikatorensatz aufzunehmen, der sich ausschließlich auf Feldvogelarten stützt und darum dem Eurostat-Vogelindex ähnelt.

\footnotetext{
${ }^{4}$ Diese Stoffe sind: Kohlendioxid, Methan, Distickstoffoxid, teilhalogenierte Fluorkohlenwasserstoffe, perfluorierte Kohlenwasserstoffe und Schwefelhexafluorid (Statistisches Bundesamt 2012: 72).
} 


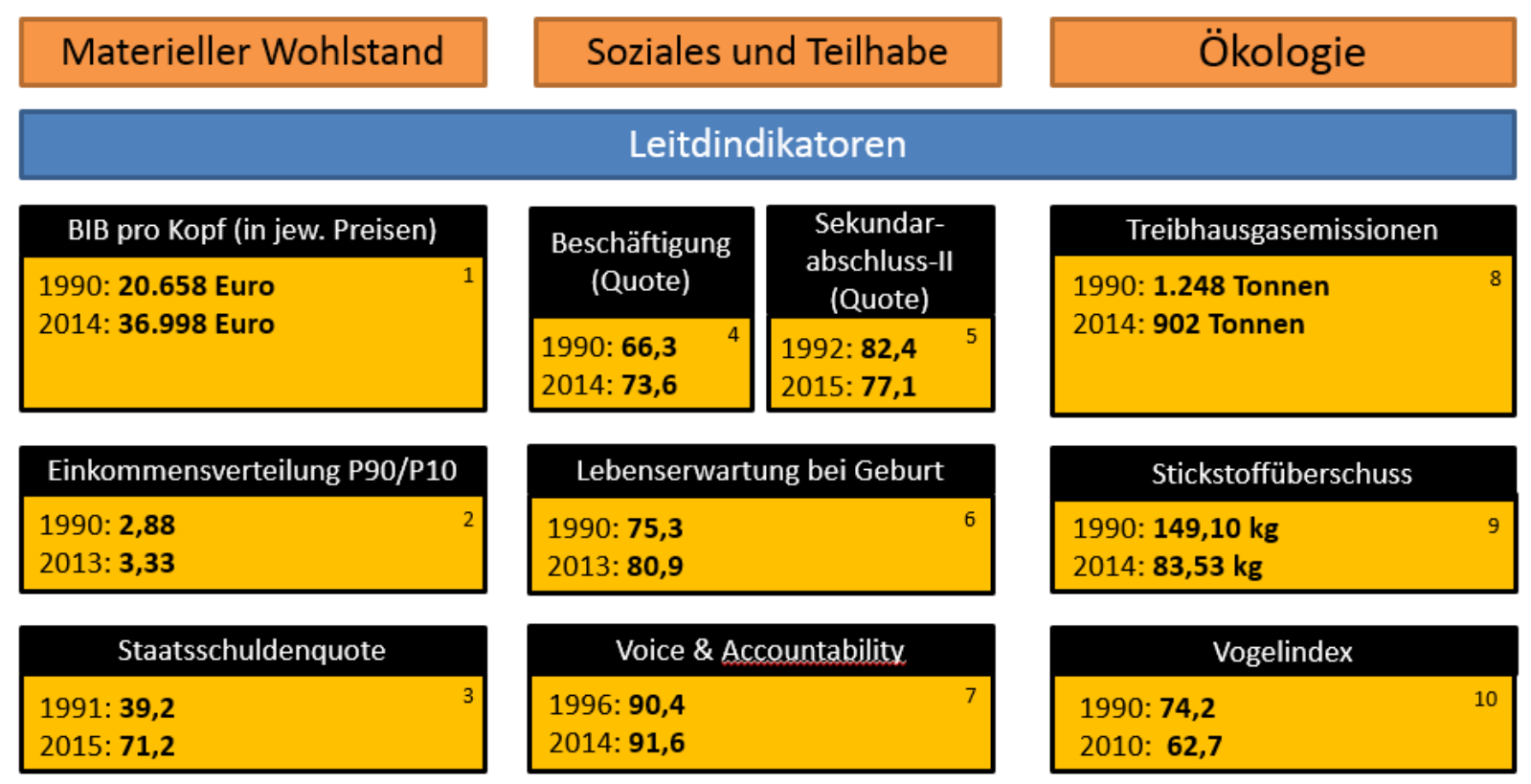

Abbildung 2: Aktuelle Werte der $W^{3}$-Leitindikatoren im Vergleich zum Beginn des Untersuchungszeitraums

Quelle: Eigene Darstellung auf Basis von Daten von Destatis, Bundesbank, SOEP, Eurostat, OECD, Weltbank, Umweltbundesamt, Bundesamt für Ernährung, Landwirtschaft und Verbraucherschutz, Bundesamt für Naturschutz. Die dargestellten Werte haben folgende Einheiten: 1) Euro; 2) Quotient; 3) Prozent des BIP; 4) Prozent der Gesamtbevölkerung; 5) Prozent der 20-24-Jährigen; 6) Jahre; 7) PercentilRanking; 8) Tonnen $\mathrm{CO}^{2}$-Äquivalent; 9) Kilogramm Stickstoff pro Hektar; 10) Prozent des Zielwerts. (Eigene Darstellung in Anlehnung an Enquete 2013: 276)

\section{Stand der Forschung zu Wohlfahrtsmessung}

Im Rahmen der wissenschaftlichen Diskussion um alternative Ansätze der Wohlfahrtsmessung existiert eine enorme Bandbreite wissenschaftlicher Arbeiten, die die Zusammenhänge der von den $\mathrm{W}^{3}$-Leitindikatoren gemessenen Phänomene untersuchen. Der folgende Abschnitt liefert einen Überblick wesentlicher Theorien und empirischer Publikationen.

\subsection{Indikatorenübergreifende Forschung}

Die OECD hat sich 2006 mit der Frage auseinandergesetzt, inwieweit die 16 Sozialindikatoren ihrer regelmäßigen Erhebung "Society at a Glance“ mit dem Bruttoinlandsprodukt pro Kopf korrelieren (Boarini et al., 2006). Mithilfe einer Korrelationsanalyse wurden sowohl Querschnitts- als auch Zeitreihendaten von maximal 1970 bis 2003 für alle OECD-Länder untersucht. Die Autoren zeigen, dass in der länderübergreifenden Niveaubetrachtung zahlreiche signifikante Korrelationen nachgewiesen werden können, während diese in der Betrachtung der 
Veränderungsraten in den Längsschnittdaten größtenteils verloren gehen. Hinsichtlich der Indikatoren, die sich so oder in ähnlicher Form auch unter den $\mathrm{W}^{3}$ Leitindikatoren finden, wurden signifikante Korrelationen in den Niveaus des „BIP pro Kopf“ und der Indikatoren „Beschäftigungsquote“, „Jahre des Schulbesuchs“, „GiniKoeffizient" und "Lebenserwartung bei Geburt" im Bereich von 0,55 bis 0,7 nachgewiesen. In der Betrachtung der Veränderungsraten bleibt lediglich eine positive Korrelation von ca. 0,45 zwischen dem „BIP pro Kopf“ und dem Indikator der „Einkommensverteilung“ (Gini-Koeffizient).

Sonja C. Kassenböhmer und Christoph M. Schmidt haben sich 2011 mit den verschiedenen Dimensionen der Wohlfahrt auseinandergesetzt, wie sie von der Stiglitz-Sen-Fitoussi-Kommission im Jahr 2009 in dem Bericht „Beyond GDP“ für die französische Regierung vorgeschlagen wurden (Kassenböhmer/Schmidt 2011: 2; Stiglitz et al. 2009: 14f). Dazu haben die Autoren zu den von Stiglitz et al. vorgeschlagenen Dimensionen konkrete Indikatoren eruiert. Sowohl auf der Mikroals auch auf der Makro-Ebene wurde auf Basis aggregierter Daten des Statistischen Bundesamtes sowie des Sozioökonomischen Panels für die Jahre 1991 bis 2008 mit Hilfe einer Hauptkomponentenanalyse untersucht, inwieweit die alternativen Wohlfahrtsindikatoren über die ökonomischen Standardindikatoren hinaus zusätzliche Informationen liefern. Ein Hauptergebnis ist, dass über ökonomische Kernindikatoren wie „BIP pro Kopf“, „Arbeitseinkommen“ und „Arbeitslosigkeit" hinaus zusätzliche Wohlfahrtsindikatoren nur bedingt dazu in der Lage sind, ein breiteres Bild der Wohlfahrt in Deutschland zu vermitteln (Kassenböhmer/Schmidt 2011: 25). Neben zahlreichen rein subjektiven Indikatoren wie der "Lebenszufriedenheit" oder „Sorgen um die Umwelt“ waren es - gerade auch mit Blick auf die W' ${ }^{3}$-Leitindikatoren - Indikatoren wie "Luftverschmutzung" (auf Makro-Ebene) und "Bildung“ (auf MikroEbene), deren zusätzlichen Informationsgehalt die Autoren in Frage stellen (Kassenböhmer/Schmidt 2011: 19-22).

Einen zentralen deutschen Beitrag zum Thema der alternativen Wohlfahrtsmessung anhand von Indikatorensätzen hat im Jahr 2010 der Sachverständigenrat zur Begutachtung der gesamtwirtschaftlichen Entwicklung (SVR) in Zusammenarbeit mit dem französischen Conseil d'Analyse économique geliefert (SVR 2010). Ebenfalls in Anlehnung an die Vorarbeit der Stiglitz-Sen-Fitoussi-Kommission (Stiglitz et al. 2009) entwickelte der Sachverständigenrat einen konkreten Vorschlag für ein Indikatorenset. Während der SVR-Bericht dezidiert auf Korrelationen des BIPs mit 
alternativen Ansätzen wie dem Human Development Index eingeht (SVR 2010: 6), spielen die Zusammenhänge unter den Einzelindikatoren des vorgeschlagenen Sets nur eine eher untergeordnete Rolle. Nichtsdestotrotz verweist der Sachverständigenrat auf einen hohen und robusten Zusammenhang zwischen dem Bildungsindikator "Lese- und Rechenfertigkeit" und Indikatoren für Beschäftigung und Gesundheit (SVR 2010: 85f.). Ebenfalls wird darauf hingewiesen, dass mangelnde Gesundheit, also sich negativ entwickelnde Gesundheitsindikatoren, Indikatoren anderer Dimensionen der Lebensqualität negativ beeinflussen (SVR 2010: 82). Konkret wird zudem auf die negativen Einflüsse von sich verschlechternden Umweltindikatoren auf die Gesundheit verwiesen (SVR 2010: 96) sowie die negative Korrelation zwischen Staatsverschuldung und Wachstum diskutiert (SVR 2010: 116). Die Erkenntnisse im SVR-Bericht basieren dabei allerdings nicht auf eigenen empirischen Analysen. Ähnlich geht der Bericht der Stiglitz-Sen-Fitoussi-Kommission vor (Stiglitz et al. 2009). Im Detail werden darin mögliche Auswirkungen verschiedener objektiv messbarer Bereiche der Lebensqualität auf das subjektive Wohlergehen besprochen (Stiglitz et al. 2009: 148). Konkrete Zusammenhänge einzelner objektiver Maße werden hingegen nur am Rande thematisiert: So wird etwa auf eine positive Korrelation zwischen Bildung und Gesundheit und (eingeschränkt) auch politischer Beteiligung (Stiglitz et al. 2009: 165) sowie auf eine negative Korrelation zwischen Umweltverschmutzung und Gesundheit verwiesen (Stiglitz et al. 2009: 188).

\subsection{Forschung zu den $\mathbf{W}^{3}$-Leitindikatorenpaaren}

Vor dem Hintergrund dieser insgesamt eher schwachen empirischen Lage lohnt es sich, einen Schritt weiter zu gehen. Wird die Diskussion nicht mehr im Rahmen von Indikatorensets geführt, sondern werden vielmehr die Zusammenhänge der einzelnen ökonomischen, sozialen und ökologischen Phänomene untersucht, wie sie auch im $\mathrm{W}^{3}$-Indikatorensatz vorkommen ${ }^{5}$, so liegt eine umfassende Literatur vor, die vielfach an Standardfragen der Volkswirtschaftslehre rührt.

Beispielhaft sei auf die umfangreiche Diskussion um den Zusammenhang von „BIP pro Kopf“ mit der „Einkommensverteilung“ verwiesen. Die von Kuznets unterstellte

\footnotetext{
${ }^{5}$ Es sei darauf hingewiesen, dass im Abschnitt 3.2 die W3-Umweltleitindikatoren wegen uneindeutiger Abgrenzung der Forschungsstände hinsichtlich einzelner Verschmutzungsarten bzw. der Artenvielfalt jeweils zu einem Block ( $W^{3}$-Umweltindikatoren) zusammengefasst wurden.
} 
glockenförmige Entwicklung der Einkommensverteilung (Kuznets, 1955: 8), die den Anstieg des Bruttoinlandsprodukts pro Kopf beim Übergang von einer agrarisch in eine industriell geprägte Gesellschaft begleitet (sog. „Kuznets-Kurve“), wurde in der jüngeren Geschichte von der auf breiter Front ansteigenden Ungleichverteilung in den Industrieländern infrage gestellt (Wilkonson/Pickett 2010: 29ff.). Jüngere Theorien erkennen die Ursachen hierfür beispielsweise in asymmetrischen Kapitalmarktzinsen für Kreditgeber/-nehmer oder in verzerrten Investitionsentscheidungen, ausgelöst durch Umverteilung (vgl. Galor 2011; Banerjee/Duflo, 2003; Barro, 2000; Perotti, 1996). Eine ganze Reihe empirischer Untersuchungen kommt zu heterogenen Ergebnissen, sodass Grömling und Schröder (2012: 150) einen Zusammenhang zwischen Wachstum und Ungleichverteilung annehmen, diesen jedoch als nicht kausal bezeichnen. Übertragen auf das entsprechende $\mathrm{W}^{3}$-Leitindikatorenpaar lässt sich damit keine eindeutige theorie- und von Empirie gestützte Prognose treffen.

Nicht minder umfangreiche Diskussionen ließen sich auch für die meisten weiteren Leitindikatorenpaare des $\mathrm{W}^{3}$-Satzes aufführen. Tabelle 1 listet alle Paare auf und gibt in verkürzter Form literaturbasierte Auskunft über die möglichen Zusammenhänge der Variablen. Hervorzuheben ist, dass die genannten Zusammenhänge oft weit weniger eindeutig sind, als es die kurze Nennung in der Tabelle impliziert. Denn in den meisten Fällen stellen sie die Mehrheitsmeinung bzw. die Quintessenz aus wissenschaftlichen Debatten dar, in der sowohl unterschiedliche theoretische Ansätze als auch heterogene empirische Ergebnisse aufeinandertreffen.

Tabelle 1: Literaturüberblick von Zusammenhängen der $\mathbf{W}^{3}$-Leitindikatoren

\begin{tabular}{|c|c|c|}
\hline W $^{3}$-Indikatorenpaar & $\begin{array}{l}\text { Möglicher } \\
\text { Zusammenh } \\
\text { ang }\end{array}$ & Ausgewählte Literatur \\
\hline $\begin{array}{l}\text { "BIP pro Kopf“ und } \\
\text { "Staatsschuldenquote" }\end{array}$ & negativ & $\begin{array}{l}\text { Checherita-Westphal/Rother (2012); } \\
\text { Kumar/Woo (2010); Szabó (2013), } \\
\text { Brügelmann (2012) }\end{array}$ \\
\hline $\begin{array}{l}\text { "BIP pro Kopf“" und } \\
\text { „Beschäftigungsquote" }\end{array}$ & positiv & Okun (1962); Mattoscio et al. (2012) \\
\hline $\begin{array}{l}\text { "BIP pro Kopf" und } \\
\text { "Sekundarabschluss-II- } \\
\text { Quote" }\end{array}$ & positiv & $\begin{array}{l}\text { Sianesi/Van Reenen (2003); } \\
\text { Hanushek/Wößmann (2010); Barro } \\
\text { (2000). }\end{array}$ \\
\hline $\begin{array}{l}\text { "BIP pro Kopf“ und } \\
\text { „Voice \& Accountability“ }\end{array}$ & unklar & $\begin{array}{l}\text { Sirowy/Inkeles (1990); Obinger (2004); } \\
\text { Kennedy (2010) }\end{array}$ \\
\hline $\begin{array}{l}\text { "BIP pro Kopf" und } \mathrm{W}^{3} \text { - } \\
\text { Umweltindikatoren }\end{array}$ & unklar & $\begin{array}{l}\text { Bardt (2012); Piaggio/Padilla (2012); } \\
\text { Stern (2004); Bengochea-Morancho et }\end{array}$ \\
\hline
\end{tabular}




\begin{tabular}{|c|c|c|}
\hline & & al. (2001); Dietz/Adger (2003) \\
\hline $\begin{array}{l}\text { "Einkommensverteilung" } \\
\text { und } \\
\text { "Staatsschuldenquote" }\end{array}$ & positiv & $\begin{array}{l}\text { Azzimonti et al. (2012); Salti (2010); } \\
\text { Agnello/Sousa (2012) }\end{array}$ \\
\hline $\begin{array}{l}\text { "Einkommensverteilung" } \\
\text { und } \\
\text { "Beschäftigungsquote" }\end{array}$ & positiv & $\begin{array}{l}\text { Biewen/Juhasz (2010); Schmidt/Stein } \\
\text { (2013) }\end{array}$ \\
\hline $\begin{array}{l}\text { "Einkommensverteilung" } \\
\text { und "Sekundarabschluss- } \\
\text { II-Quote" }\end{array}$ & unklar & $\begin{array}{l}\text { Rodríguez-Pose/Tselios (2009); } \\
\text { Blanden/Machin (2010); Machin/Van } \\
\text { Reenen (2008); Gregorio/Lee (2002); } \\
\text { Földvári/Leeuwen (2011) }\end{array}$ \\
\hline $\begin{array}{l}\text { "Einkommensverteilung“ } \\
\text { und "Lebenserwartung } \\
\text { bei Geburt" }\end{array}$ & negativ & $\begin{array}{l}\text { Wilkinson/Pickett (2010); Cantarero et } \\
\text { al. (2005); Kibele et al. (2013) }\end{array}$ \\
\hline $\begin{array}{l}\text { "Einkommensverteilung" } \\
\text { und "Voice \& } \\
\text { Accountability" }\end{array}$ & unklar & $\begin{array}{l}\text { Acemoglu/Robinson (2006); Houle } \\
\text { (2009); Yi (2012) }\end{array}$ \\
\hline $\begin{array}{l}\text { "Einkommensverteilung" } \\
\text { und } \mathrm{W}^{3} \text { - } \\
\text { Umweltindikatoren }\end{array}$ & $\begin{array}{l}\text { Treibhausgas } \\
\text { emissionen } \\
\text { und } \\
\text { Stickstoffüber } \\
\text { schuss: } \\
\text { unklar } \\
\text { Vogelartenind } \\
\text { ex: positiv }\end{array}$ & $\begin{array}{l}\text { Heerink et al. (2001); Baek/Guankerwon } \\
\text { (2013); Mikkelsen et al. (2007); Holland } \\
\text { (2009) }\end{array}$ \\
\hline $\begin{array}{l}\text { "Staatsschuldenquote" } \\
\text { und } \\
\text { "Beschäftigungsquote" }\end{array}$ & negativ & Coccia (2012) \\
\hline $\begin{array}{l}\text { "Staatsschuldenquote" } \\
\text { und "Lebenserwartung } \\
\text { bei Geburt"; } \\
\text { "Sekundarabschluss-II- } \\
\text { Quote“, „Voice \& } \\
\text { Accountability“, W" } \\
\text { Umweltindikatoren }\end{array}$ & - & keine hinreichende Forschung bekannt \\
\hline $\begin{array}{l}\text { "Beschäftigungsquote“ u } \\
\text { nd „Sekundarabschluss- } \\
\text { II-Quote }\end{array}$ & positiv & $\begin{array}{l}\text { Ridell/Song (2011); Hérault et al. (2012); } \\
\text { OECD (2013b) }\end{array}$ \\
\hline $\begin{array}{l}\text { "Beschäftigungsquote" } \\
\text { und "Voice \& } \\
\text { Accountability" sowie W' }{ }^{3}- \\
\text { Umweltindikatoren }\end{array}$ & - & Keine hinreichende Forschung bekannt \\
\hline $\begin{array}{l}\text { „Sekundarabschluss-II- } \\
\text { Quote“ und } \\
\text { "Lebenserwartung bei } \\
\text { Geburt“ }\end{array}$ & positiv & $\begin{array}{l}\text { Muening (2010); Eide/Showalter (2010); } \\
\text { Hazan (2012); Schneider (2007) }\end{array}$ \\
\hline $\begin{array}{l}\text { "Sekundarabschluss-II- } \\
\text { Quote“ und „Voice \& } \\
\text { Accountability" }\end{array}$ & positiv & $\begin{array}{l}\text { McMahon (2010); Dee (2004); Glaeser } \\
\text { et al. (2007) }\end{array}$ \\
\hline $\begin{array}{l}\text { "Sekundarabschluss-II- } \\
\text { Quote" und W" }{ }^{3} \text { - }\end{array}$ & unklar & $\begin{array}{l}\text { Torras/Boyce (1998); Kinda (2010); } \\
\text { Jorgenson (2003) }\end{array}$ \\
\hline
\end{tabular}




\begin{tabular}{|c|c|c|}
\hline Umweltindikatoren & & \\
\hline $\begin{array}{l}\text { "Lebenserwartung bei } \\
\text { Geburt" und „Voice \& } \\
\text { Accountability“ }\end{array}$ & positiv & $\begin{array}{l}\text { Franco et al. (2004); Besley/Kudamatsu } \\
\text { (2006); Lora/Olivera (2007) }\end{array}$ \\
\hline $\begin{array}{l}\text { "Lebenserwartung bei } \\
\text { Geburt" und } \mathrm{W}^{3} \text { - } \\
\text { Umweltindikatoren }\end{array}$ & $\begin{array}{l}\text { unklar, da } \\
\text { Wirkungszus } \\
\text { ammenhänge } \\
\text { sehr komplex } \\
\text { und } \\
\text { zeitverzögert }\end{array}$ & $\begin{array}{l}\text { McMichael (2013); McMichael/Lindgren } \\
\text { (2011); Campell et al. (2007); Haines } \\
\text { (2012) }\end{array}$ \\
\hline $\begin{array}{l}\text { „Voice and } \\
\text { Accountability“ und W³- } \\
\text { Umweltindikatoren }\end{array}$ & positiv & $\begin{array}{l}\text { Farzanegan/Markwardt (2012); } \\
\text { Torras/Boyce (1998) }\end{array}$ \\
\hline $\begin{array}{l}\text { "Treibhausgasemissione } \\
\text { n" und "Vogelindex" }\end{array}$ & $\begin{array}{l}\text { unklar, da } \\
\text { Wirkungszus } \\
\text { ammenhänge } \\
\text { sehr komplex } \\
\text { und } \\
\text { zeitverzögert }\end{array}$ & Thuiller (2007); IPPC (2007) \\
\hline $\begin{array}{l}\text { "Treibhausgasemissione } \\
\text { n“ und } \\
\text { „Stickstoffüberschuss“ }\end{array}$ & - & Keine hinreichende Forschung \\
\hline $\begin{array}{l}\text { "Stickstoffüberschuss" un } \\
\text { d „Vogelindex“ }\end{array}$ & negativ & Diese (2011); Stevens et al. (2004) \\
\hline
\end{tabular}

Quelle: Eigene Darstellung

\section{Empirische Analyse: Verknüpfungen der $\mathbf{W}^{3}$-Leitindikatoren}

\subsection{Daten und Datenaufbereitung für Deutschland und die EU}

Da die $\mathrm{W}^{3}$-Leitindikatoren ihren primären geografischen Anwendungsbereich in Deutschland finden, besteht der erste Datensatz aus Längsschnittdaten für die Bundesrepublik Deutschland von 1990 bis 2012. Die Anzahl der Beobachtungen schwankt variablenspezifisch zwischen 14 und 23. Für neun von zehn Variablen liegen jedoch mind. 20 Beobachtungen vor (vgl. Tabelle A1 im Annex). Die Indikatoren werden in ihrer Rohversion in unterschiedlichen Einheiten gemessen und weisen durchwegs klar erkennbare Trendkomponenten auf (Becketti 2013: 93ff.). Im Fall von zwei Indikatoren („Voice \& Accountability“, „Sekundar-Abschluss-II-Quote“) weisen die Zeitreihen Lücken auf. ${ }^{6}$

Der zweite Datensatz besteht aus Querschnittsdaten der 28 EU-Länder für das Jahr 2008, die einzelnen Variablen verfügen jeweils über eine Beobachtungsanzahl

\footnotetext{
${ }^{6}$ Aufgrund der Datenverfügbarkeit sowie der Übersichtlichkeit wird pro Indikatorenbereich der zentrale Leitindikator ausgewählt. So wird lediglich die Veränderungsrate des „BIP-pro-Kopf“ betrachtet. Analog wird beim Leitindikator „Staatsschuldenquote“ die „Fiskalische Nachhaltigkeitslücke“ nicht untersucht .
} 
zwischen 15 und 28 Beobachtungen, wobei acht von zehn Variablen mindestens 26 Beobachtungen aufweisen (vgl. Tabelle A2 im Annex).

Bis auf wenige Ausnahmen stammen die Daten vom Statistischen Bundesamt, der Bundesbank, von Eurostat, der OECD und der Weltbank (Deutsche Bundesbank 2013; Weltbank 2013; Statistisches Bundesamt 2013a-C; OECD 2013a; Eurostat 2013a-j; BMELV 2016). Schwierig gestaltete sich die Recherche nach den Daten der Zeitreihe des Indikators Verteilung (P80/P20) und Artenvielfalt (Index Artenvielfalt und Landschaftsqualität, Teilindex Agrarland). Aufgrund der besseren Verfügbarkeit wird stattdessen der regelmäßig publizierte Indikator des P90/P10Einkommensverhältnisses herangezogen (DIW 2013). Die Daten zur Artenvielfalt (Index der Feldvogelarten) werden vom Bundesamt für Naturschutz zur Verfügung gestellt (Bundesamt für Naturschutz 2013).

Zur Beurteilung von Redundanzen innerhalb der $\mathrm{W}^{3}$-Indikatoren werden eine Korrelations- sowie eine Hauptkomponentenanalyse durchgeführt. Dazu werden die Daten nach dem Muster:

$$
z_{i j}=\frac{x_{i j}-\bar{x}_{j}}{s_{j}}, i=1,2, \ldots, p
$$

z-standardisiert (Voß et al. 2004: 533f.). Es werden die Abweichungen der beobachteten Werte $x_{i j}$ vom arithmetischen Mittel $\bar{x}_{j}$ der j-ten Variablen durch ihre Standardabweichung geteilt. Dies ist notwendig, da faktorenanalytische Ergebnisse sensitiv auf Veränderungen der Maßeinheiten reagieren. Das Verfahren bietet den Vorteil, dass alle Variablen auf ein einheitliches Maß gebracht werden und einen Mittelwert von 0 sowie eine Standardabweichung von 1 erhalten (OECD 2008: 28).

Bei den ursprünglichen Längsschnittdaten handelt es sich ausschließlich um Zeitreihen, die durchweg von klaren Trends gekennzeichnet sind. Durch die Bildung der ersten Differenz in jeder Zeitreihe wird die Trend-Komponente aus der Zeitreihe entfernt, dargestellt werden nun die absoluten Veränderungen zur jeweiligen Vorperiode. Der Verlust einer Beobachtung wird in Kauf genommen, damit die Zeitreihen (schwach) stationär werden (Becketti 2013: 208f., 379). Die Ergebnisse des erweiterten Dickey-Fuller Tests und in einem Fall (Neunzig-Zehn-Verteilung) des 
Phillips-Perron-Einheitswurzeltests ${ }^{7}$ belegen die (schwache) Stationarität (zum konkreten Vorgehen siehe: Becketti 2013: 381f.).

In zwei der neun betrachteten Zeitreihen wurden fehlende Werte mittels linearer Interpolation aufgefüllt (Stata 2005: 213ff.). In der Zeitreihe „Demokratie“ sind das die Werte für die Jahre 1997, 1999 und 2001. Für die Variable „Bildung“ handelt es sich um den Wert für das Jahr 1998. Die Ergebnisse von Korrelations- und Hauptkomponentenanalyse reagieren darauf allerdings nicht sensitiv. Die Datenlücken im Querschnittsdatensatz wurden nicht aufgefüllt.

In beiden Datensätzen werden die meisten Indikatoren in Quoten dargestellt, die in Prozent angegeben werden. Ausnahmen sind der Stickstoff-Indikator (Überschüsse in Kilo pro Flächeneinheit) und "Lebenserwartung bei Geburt" (Jahre). Allein der Indikator "Treibhausgasemissionen" wird bei den $\mathrm{W}^{3}$-Indikatoren unmittelbar in seinem Gesamtausstoß in Tonnen $\mathrm{CO}_{2}$-Äquivalente gemessen. Damit ergibt sich ein Ungleichgewicht in der Betrachtung, das die Ergebnisse verzerren würde. Dem wird damit begegnet, dass die Treibhausgasemissionen als Quote zur Bevölkerungszahl dargestellt und folglich die Treibhausgasemissionen pro Kopf zur Grundlage der Berechnungen werden.

\subsection{Korrelationen und Hauptkomponentenanalyse der $\mathbf{W}^{3}$-Leitindikatoren}

Für beide Datensätze werden die jeweiligen Korrelationen unter allen möglichen Variablenpaaren berechnet. Dazu wird der Korrelationskoeffizient nach BravaisPearson angewandt (Voß 2004: 187f). Eine Einschränkung der Korrelationsanalyse ist es, dass damit lediglich unmittelbare (Inter-)Dependenzen ermittelt werden, nicht aber zeitverzögerte Korrelationen. Dies wäre vor allem vor dem Hintergrund relevant, dass sich sachlogisch manche Variablen mit einiger Verzögerung an andere anpassen und nicht parallel reagieren dürften - es sei denn, sie werden von derselben, dritten Variable beeinflusst. Diese muss jedoch nicht unbedingt Teil des Indikatorensets sein. Um solche unbeobachteten, jedoch einflussreichen Variablen zu berücksichtigen, liefert die Faktorenanalyse der Hauptkomponenten (im Folgenden: „Hauptkomponentenanalyse“) teilweise eine Kompensation (Eckey

\footnotetext{
${ }^{7}$ Eine Alternative zu diesem Vorgehen im Fall der Neunzig-Zehn-Verteilung wäre eine Logarithmierung der Ursprungsdaten gewesen. Bei diesem Vorgehen erweist sich die Zeitreihe wie alle anderen bereits beim erweiterten Dickey-Fuller-Test als stationär (auf dem 10-Prozent-SN). Da die Ergebnisse der Korrelations- und der Hauptkomponentenanalyse jedoch nicht sensitiv auf die Logarithmierung des Indikators reagieren und zudem der Phillips-Perron-Test Stationarität bereits für die nicht logarithmierte Zeitreihe nahegelegt hat, wurde darauf verzichtet.
} 
2002: 5f.). Dabei handelt es sich um ein Verfahren zur Datenreduktion in der multivariaten Statistik, das dazu in der Lage ist, Faktoren nachzuweisen, die nicht Teil der im Datensatz befindlichen Variablen sind. Diese Faktoren sind als erklärende Größen hinter der Abhängigkeitsstruktur der beobachteten Variablen aufzufassen. Hängt folglich ein Verbund von Variablen eng mit einem unbeobachteten Faktor zusammen, so lässt sich daraus schließen, dass die Betrachtung des Faktors ausreicht. Die jeweiligen Variablen müssen in diesem Fall nicht mehr im Einzelnen beobachtet werden; auf sie kann in einem Indikatorensatz verzichtet werden.

Die Struktur der Abhängigkeit zwischen den Faktoren und Variablen lässt sich folgendermaßen formalisieren (Voß 2004: 533):

$$
X_{j}=\alpha_{j 1} \cdot F_{1}+\alpha_{j 2} \cdot F_{2}+\cdots+\alpha_{j m} \cdot F_{m}+U_{j}, \quad j=1,2, \ldots, p
$$

Es werden $p$ manifeste Variablen auf $m$ gemeinsame Faktoren $(m<p)$ zurückgeführt. In dieser Gleichung wird die manifeste Variable $X_{j}$ durch die gemeinsamen Faktoren $F_{1}, F_{2}, \ldots, F_{m}$ und einen merkmalsspezifischen Faktor $U_{j}$ (Residualgröße) erklärt. Die Koeffizienten $\alpha_{j m}$ heißen Faktorladungen. Stellen die einzelnen Faktoren voneinander unabhängige Dimensionen dar, so lassen sich die Faktorladungen als Korrelationskoeffizienten zwischen den betrachteten Variablen und den Faktoren interpretieren. Da sich die Faktoren nicht unmittelbar beobachten lassen, können auch die Faktorladungen nicht unmittelbar bestimmt werden (Voß 2004: 534, Eckey et al 2002: 9ff.). Um dieses Problem zu lösen, wird hier die Korrelationsmatrix $\mathrm{R}$ der beobachtbaren Variablen herangezogen, die sich in Anbetracht der z-Standardisierung (siehe oben) und unter Zuhilfenahme der Beziehung $R_{p x p}=\frac{1}{n-1} \cdot\left(Z^{\prime} Z\right)_{\text {pxnnxp }}$ mit folgender Beobachtungsmatrix $Z$ bilden lässt:

$$
Z_{n x p}=\left(\begin{array}{ccc}
z_{11} & \cdots & z_{1 p} \\
\vdots & \ddots & \vdots \\
z_{n 1} & \cdots & z_{n p}
\end{array}\right)
$$

Dabei ist $Z$ als die $z$-standardisierte Form der Variablenmatrix $X$ aufzufassen; $Z$ ist die transponierte Matrix ${ }^{8}$. Anschließend wird $Z$ über die Summe der Varianz aller Variablen analysiert, die einen Beitrag zu einem der Faktoren liefern (Kassenböhmer/Schmidt 2010: 11ff.). Durch eine Hauptkomponentenanalyse der Korrelationsmatrix (ohne den unerklärten Teil der Varianz) ergeben sich die 
Eigenwerte, die die Faktorladungen $\alpha_{j m}$ bestimmen. Eingang in die Betrachtung finden ausschließlich Faktoren, deren Eigenwerte größer 1 sind (sog. „KaiserKriterium", Voß 2004: 544).

Im nächsten Schritt werden diejenigen Variablen bestimmt, auf die diese Faktoren am höchsten laden (Kassenböhmer/Schmidt 2010: 12, 17f.). Auf sie wird das Gesamtsample reduziert. Hier unterscheidet sich das Vorgehen in dieser Arbeit von der klassischen Hauptkomponentenanalyse, die auf die Faktoren selbst abstellt. Statt der Faktoren selbst werden die Variablen im Set behalten, die jeweils am höchsten auf die einzelnen Faktoren laden. Diejenigen, die weniger hoch auf dieselben Faktoren laden, werden nicht länger berücksichtigt. Mit dieser Reduzierung des Indikatorensatzes geht ein Informationsverlust einher. Um zu beurteilen, wie groß dieser ist, werden die ausgewählten Variablen erneut einer Hauptkomponentenanalyse unterzogen. Korrelieren die Faktoren dieses reduzierten Indikatorensatzes stark mit denen des Gesamt-Samples, so ist der Informationsverlust überschaubar. Zur Beurteilung, ob sich die Variablen für eine Hauptkomponentenanalyse eignen, wurde das Kaiser-Meyer-Olkin-Kriterium (KMO für das Gesamtsample/kmo für einzelne Variablen) geprüft (Eckey et al. 2002: 20).

Eine Anwendung der Hauptkomponentenanalyse für die EU-Daten würde bedeuten, dass die Ergebnisse für den zeitlichen Ablauf in Deutschland denen für die NiveauUnterschiede im EU-Ausland gegenübergestellt werden. Um dieses Problem, auf das auch der Sachverständigenrat für Gesamtwirtschaftliche Entwicklung hingewiesen hat, zu umgehen, wird die Methode nur auf den nationalen Zeitreihen-Datensatz angewandt.

\subsection{Optimale Anzahl der $\mathbf{W}^{3}$-Leitindikatoren}

Es werden anhand der beiden Datensätze 45 Zweier-Kombinationen der zehn $\mathrm{W}^{3}$ Leitindikatoren auf den Korrelationskoeffizienten nach Bravais-Pearson geprüft. Ziel ist es, die Korrelation jedes Indikators mit jedem weiteren einmal zu berechnen. Die Tabellen 2 und 3 zeigen alle statistisch signifikanten Korrelationen (auf dem 10- und dem 5-Prozent-Signifikanzniveau). Anhand der Deutschland-Daten weisen sieben der 45 Leitindikatorenpaare signifikante Korrelationen auf; sechs davon auf dem 5Prozent-Niveau, eine auf dem 10-Prozent-Niveau. Sie bewegen sich im Bereich von $|0,41|$ und $|0,67|$. Der EU-28-Datensatz erbrachte eine signifikante Korrelation von 20 der 45 Paare, 18 davon auf einem Signifikanz-Niveau von 5 Prozent, zwei auf dem 
10-Prozent-Niveau. Die Stärke der Korrelationen bewegt sich in einer Spannweite von $|0,37|$ und $|0,79|$. Vier der sechs Korrelationen, die in den Zeitreihen für Deutschland gefunden werden, finden sich auch auf der internationalen EU-Ebene.

Tabelle 2: Darstellung der signifikanten Korrelationen der Längsschnittdaten $\left(10 \% ; 5 \%{ }^{*}\right)$

\begin{tabular}{|c|c|c|c|c|c|c|c|c|c|c|}
\hline & bip̃ pc & neun zehn & schulden & beschaef & bildung & $\begin{array}{l}\text { lebens } \\
\sim\end{array}$ & demokr & treibh & $\begin{array}{l}\text { stickst } \\
\end{array}$ & vogel \\
\hline bip_real_pc & 1.000 & & & & & & & & & \\
\hline neunzig_zehn & & 1.000 & & & & & & & & \\
\hline schuldenquote & $-0.546^{*}$ & & 1.000 & & & & & & & \\
\hline beschaeftig & $0.550^{*}$ & & -0.406 & 1.000 & & & & & & \\
\hline bildung & & & & & 1.000 & & & & & \\
\hline lebenserw & & $-0.508^{*}$ & & & & 1.000 & & & & \\
\hline demokratie & & & & & & & 1.000 & & & \\
\hline treibhausg & $0.450^{*}$ & $0.471^{*}$ & & & & & & 1.000 & & \\
\hline stickstoff & 0.674 & & & & & & & & 1.000 & \\
\hline vogelindex & & & & & & & & & & 1.000 \\
\hline
\end{tabular}

Tabelle 3: Darstellung der signifikanten Korrelationen der Querschnittsdaten (10\%; 5\%*)

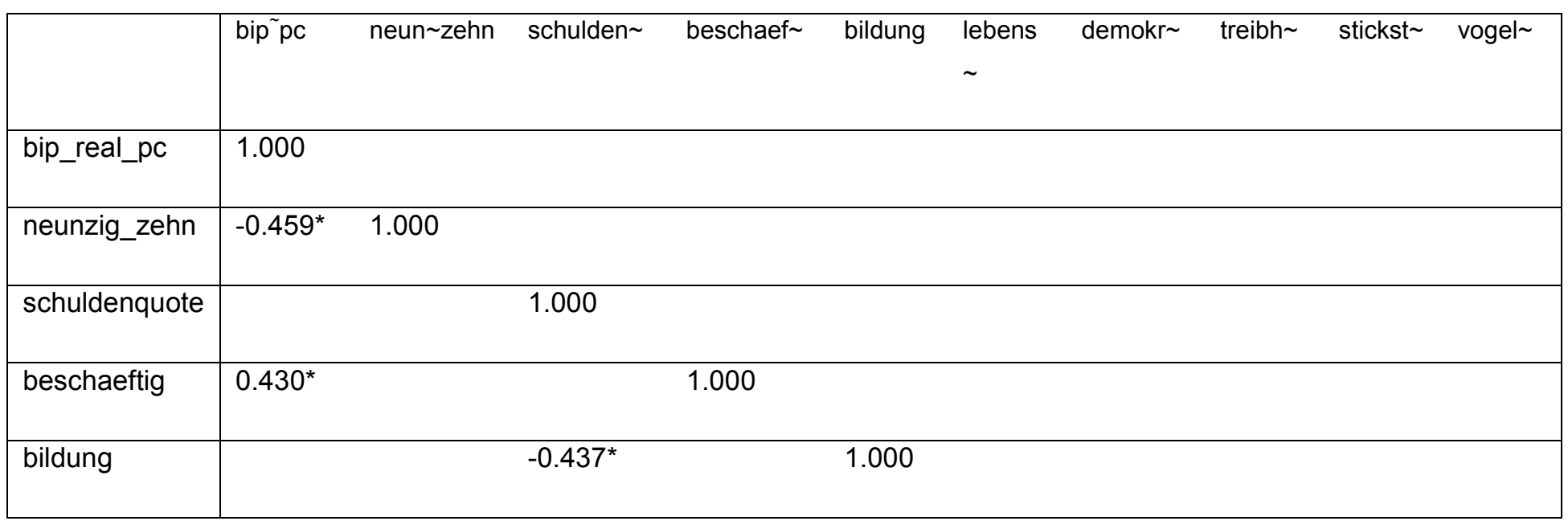




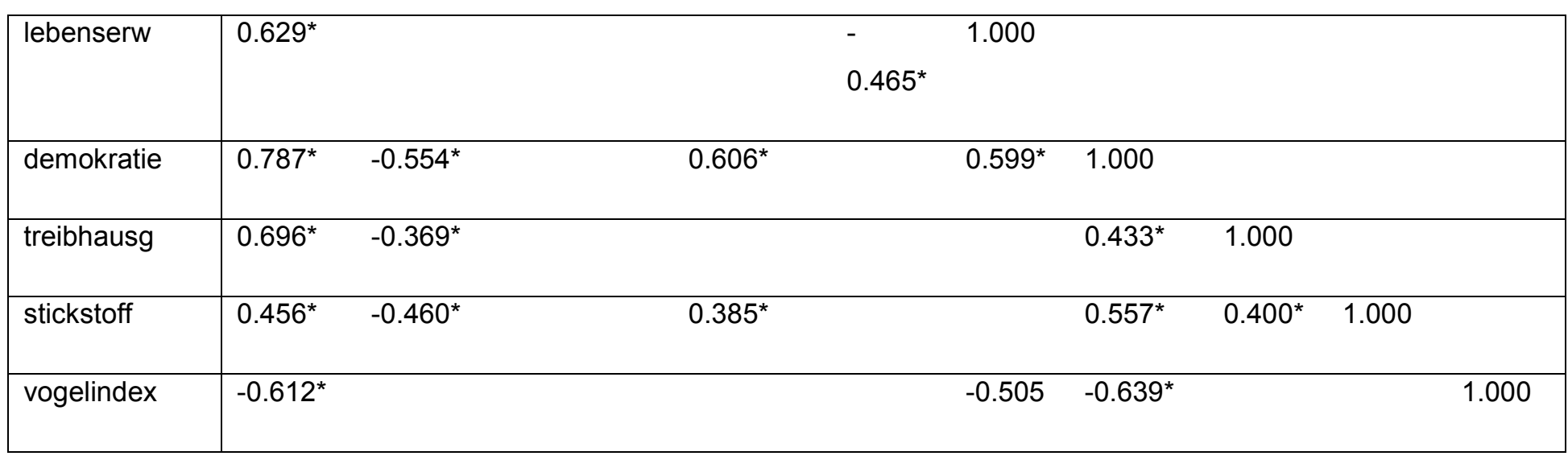

Quelle: Eigene Berechnungen und Darstellung.

Damit bestätigt sich ein erster empirischer Befund, zu dem auch Boarini et al. (2006: 30) kommen: Während in der länderübergreifenden Niveau-Betrachtung zahlreiche Korrelationen festgestellt werden können, gehen die meisten davon verloren, sobald sie über Veränderungsraten nachgewiesen werden sollen. Analog bilden die EUDaten das jeweils länderspezifische Niveau der $W^{3}$-Indikatoren im Jahr 2008, die Deutschland-Daten nehmen die Differenz zum Vorjahr in den Blick. Im Folgenden werden nun die signifikanten Korrelationen innerhalb der deutschen Zeitreihendaten mit den Ergebnissen der EU-Querschnittsdaten sowie früherer Publikationen verglichen, um anschließend eine erste Aussage über den Verbleib bzw. Ausschluss der korrelierten Leitindikatoren zu treffen.

Anhand der Zeitreihen im Deutschland-Datensatz zeigt sich eine signifikant negative Korrelation mittlerer Stärke (-0,55/5-Prozent-SN) zwischen den beiden $\mathrm{W}^{3}$ Leitindikatoren „Bruttoinlandsprodukt pro Kopf“ (bip_real_pc) und "Staatsschuldenquote“ (schuldenquote). Ein Anstieg des BIP pro Kopf geht tendenziell mit einer sinkenden Staatsschuldenquote einher et vice versa. Zwar wird dieses Ergebnis nicht von den EU-Daten gestützt, doch legt die eindeutige theoretische sowie empirische Lage eine Korrelation für Deutschland nahe. Zudem ist sie international nachgewiesen worden (u. a. Coccia 2012, ChecheritaWestphal/Rother 2012). Aufgrund der Korrelation lässt sich schließen, dass der W³Indikator „Staatsschuldenquote“ - zumindest teilweise - redundant ist.

Ebenso ist die Korrelation zwischen den beiden $\mathrm{W}^{3}$-Leitindikatoren „Bruttoinlandsprodukt pro Kopf“ und „Beschäftigungsquote“ (beschaeftigung) in den Deutschlanddaten signifikant (+0,55/5-Prozent SN). Das heißt, wenn das BIP pro Kopf steigt, dann steigt tendenziell auch die Beschäftigungsquote et vice versa. 
Diese Korrelation findet sich auch in der Analyse der EU-28-Niveaudaten $(+0,43 / 5-$ Prozent-SN). Die Korrelation kann über das „Okun'sche Gesetz“ erklärt werden, das eine Senkung der Arbeitslosigkeit (und damit - zumindest in der Regel - implizit eine Steigerung der Beschäftigung) bei steigendem Bruttoinlandsprodukt vorhersagt (Okun 1962: 102, Blanchard-Fisher 1993: 364f., Mankiw 2010: 260ff.). Empirisch wurde der Zusammenhang in der Vergangenheit vielfach nachgewiesen (u. a. Mattoscio 2012: 242). Folglich ist der $W^{3}$-Leitindikator „Beschäftigungsquote“ zumindest teilweise - ebenfalls redundant.

Eine signifikant positive Korrelation gibt es zwischen dem „Bruttoinlandsprodukt pro Kopf“ und den beiden $W^{3}$-Umweltindikatoren „Treibhausgasemissionen“ (treibhausg) (+0,45/5-Prozent-SN) und „Stickstoffüberschuss“ (stickstoff) (+0,67/5-Prozent-SN) für die deutschen Zeitreihendaten. Diese Ergebnisse werden von den EU-Querschnittsdaten gestützt. Hinsichtlich der realen Entwicklung der Treibhausgasemissionen konnte im Untersuchungszeitraum zwar eine relative und absolute Entkopplung vom Wirtschaftswachstum erreicht werden (Bardt 2012: 103). Wie auch das Ergebnis zeigt, bedeutet das jedoch nicht, dass ein Anstieg des BIPs nicht mehr mit zusätzlichen Emissionen verbunden ist.

Empirisch wird ein positiver Zusammenhang zwischen Kohlendioxidausstoß und BIP beispielsweise von Bengochea-Morancho et al. (2001: 168) für zehn EU-Staaten nachgewiesen und in einer Meta-Studie von David I. Stern nahegelegt (Stern 2004: 1435). Angesichts der relativ starken Korrelation der Indikatoren „BIP pro Kopf“ und "Stickstoffüberschuss" auf deutscher Ebene (und der Tatsache, dass diese von internationalen Daten gestützt wird), scheint der $W^{3}$-Leitindikator „Stickstoffüberschuss“ keinen großen Erkenntnisgewinn zu bescheren und kann deshalb aus dem Indikatorensatz entfernt werden. Die auf deutscher Ebene schwächere Korrelation der Leitindikatoren „BIP pro Kopf“ und „Treibhausgasemissionen“ rechtfertigt hingegen einen Verbleib dieser beiden Indikatoren im Set.

Der Leitindikator „Treibhausgasemissionen“ ist nicht nur mit dem „Bruttoinlandsprodukt pro Kopf“, sondern auch mit dem Leitindikator für „Einkommensverteilung“ (neunzig_zehn) statistisch signifikant schwach positiv korreliert $(+0,47 / 5$-Prozent-SN). Sinkt in Deutschland die Ungleichverteilung des 
Einkommens über die Zeit, so sinken in der Tendenz auch die „Treibhausgasemissionen“. Dieses Ergebnis wird nicht von den EU-Daten gestützt. Ein möglicher Erklärungsansatz für die deutschen Ergebnisse ist die politökonomische These, dass in Gesellschaften mit gleicher verteiltem Einkommen ein größerer politischer Druck hinsichtlich einer besseren Umweltqualität ausgeübt wird (Heerink et al. 2001: 360). Die lediglich schwache positive Korrelation beider Leitindikatoren rechtfertigt jedoch den Verbleib dieser beiden Größen in den $\mathrm{W}^{3}$ Indikatoren.

Die letzte statistisch signifikante Korrelation im $\mathrm{W}^{3}$-Leitindikatorenset auf 5-ProzentSignifikanzniveau besteht zwischen den Indikatoren „Einkommensverteilung“ und „Lebenserwartung bei Geburt“ (lebenserw) (-0,52). Die Korrelation hat eine mittlere Stärke und ist negativ. Das bedeutet, dass eine steigende Ungleichverteilung der Einkommen in Deutschland mit einer sinkenden Lebenserwartung bei Geburt einhergeht. Diese Korrelation findet sich zwar nicht in den EU-Daten, dafür allerdings in zahlreichen früheren empirischen Arbeiten. Alle gründen darauf, dass sich ungleicher verteiltes Einkommen negativ auf die psychosozialen Determinanten der Gesundheit auswirkt und darum einen früheren Tod wahrscheinlicher macht. Folglich liegt der Schluss nahe, dass auf den Indikator „Lebenserwartung bei Geburt“ als W³Leitindikator verzichtet werden kann.

Lediglich bei 10-Prozent signifikant ist für Deutschland die Korrelation zwischen den Leitindikatoren „Staatsschuldenquote“ und „Beschäftigungsquote“ (-0,41/10Prozent-SN). In den EU-Daten ist dieser Zusammenhang zwar ebenfalls zu finden ($0,22)$, allerdings ist er hier nicht signifikant. Dieser Effekt kann indirekt über den theoretischen Nexus zwischen „Staatsschuldenquote“ und Wirtschaftswachstum (also Veränderungsraten des BIP pro Kopf) erklärt werden: Steigt die Staatsverschuldung, so leidet darunter in der Tendenz das Wirtschaftswachstum (z. B. durch „Crowding Out privater Investitionen“) (vgl. Reinhard/Rogoff 2010; Herndon et al. 2013: 13; Brügelmann 2012: 123) und in der Folge auch die Beschäftigung. Aufgrund der sowohl schwachen Signifikanz als auch dem relativ geringen Korrelationsniveau scheint eine Entfernung der „Staatsschuldenquote“ aus den $\mathrm{W}^{3}$ Leitindikatoren jedoch mit einem zu großen Erkenntnisverlust einherzugehen.

Damit lautet das Ergebnis der Korrelationsanalyse, dass sich der Satz aus zehn W³Leitindikatoren ohne großen Informationsverlust auf sechs Leitindikatoren hätte 
reduzieren lassen. Die Indikatoren "Stickstoffüberschuss", „Staatsschuldenquote“, „Beschäftigungsquote" und "Lebenserwartung bei Geburt" sind allesamt deutlich mit den Leitindikatoren "Bruttoinlandsprodukt pro Kopf“ sowie „Einkommensverteilung“ korreliert. Dieses Ergebnis wird entweder von den EUQuerschnittsdaten oder von den Ergebnissen vorangegangener Publikationen validiert. Deshalb ginge ihre Entfernung kaum mit Erkenntnisverlusten über den Zustand der Wohlfahrt in Deutschland einher.

Die Hauptkomponentenanalyse untersucht die $W^{3}$-Leitindikatoren, indem sie die Korrelationen betrachtet, die nicht ausschließlich unter den beobachteten Indikatorenpaaren bestehen, sondern die von mehreren Leitindikatoren geteilt werden und so auf dahinterliegende, unbeobachtete Phänomene (Faktoren) verweisen. Im Folgenden wird untersucht, ob sich die Ergebnisse der Korrelationsanalyse über die Hauptkomponentenanalyse stützen lassen.

Die Analyse zeigt, dass hinter den zehn $\mathrm{W}^{3}$-Leitindikatoren insgesamt vier (unbeobachtete) Faktoren stehen, deren Eigenwert gemäß dem Kaiser-Kriterium größer eins ist (Tabelle 4). Zusammen erklären diese vier Faktoren 73,22 Prozent der Gesamtvariation aller Variablen.

Tabelle 4 Eigenwertbetrachtung der Faktoren des gesamten Samples

\begin{tabular}{|l|cccc|}
\hline Factor & Eigenvalue & Difference & Proportion & Cumulative \\
\hline Factor 1 & 2.869 & 1.020 & 0.287 & 0.287 \\
Factor 2 & 1.849 & 0.396 & 0.185 & 0.472 \\
Factor 3 & 1.453 & 0.301 & 0.145 & 0.617 \\
Factor 4 & 1.152 & & 0.115 & 0.732 \\
\hline
\end{tabular}

Die Hauptkomponentenanalyse ergibt, dass hinter den zehn $\mathrm{W}^{3}$-Leitindikatoren insgesamt vier unbeobachtete Faktoren stehen, die gemäß Kaiser-Kriterium (Eigenwert $>1$ ) in die Betrachtung aufgenommen werden können (Eigene Berechnung und Darstellung).

Tabelle 5 zeigt, wie hoch die vier Faktoren auf die einzelnen Variablen laden; aufgeführt werden im Folgenden diejenigen, deren Faktorladung größer dem Schwellenwert 0,5 ist. Die höchste Faktorladung des Faktors 1 entfällt auf das „BIP pro Kopf" $(+0,95)$; ebenfalls hoch lädt dieser Faktor auf die Indikatoren „Beschäftigungsquote“ $(+0,62)$, „Bildung“ $(+0,62)$, „Treibhausgasemissionen“ $(+0,65)$ und "Stickstoffüberschuss" $(+0,58)$. Kein anderer Faktor lädt auf diese Variablen 
höher. Die höchsten Faktorladungen des Faktors 2 entfallen auf die Variablen "Lebenserwartung" (-0,79), „Einkommensverteilung" $(+0,78)$ und erneut Sticktstoffüberschuss $(+0,58)$. Die höchste Faktorladung des Faktors 3 entfällt auf die Demokratie-Variable "Voice \& Accountability" $(-0,92)$, die zweithöchste auf die Variable "Staatsschuldenquote" $(+0,64)$. Die höchste Faktorladung des Faktors 4 entfällt auf den Artenvielfalts-Indikator „Vogelindex“ $(+0,79)$. Keine weitere Variable wird vom Faktor 4 durch eine hohe Faktorladung repräsentiert.

Tabelle 5 Die Faktoren und ihre Faktorladungen auf die Variablen

\begin{tabular}{|l|cccc|c|}
\hline Variable & Factor 1 & Factor 2 & Factor 3 & Factor 4 & Uniqueness \\
\hline bip_real_pc & 0.954 & -0.018 & -0.101 & -0.123 & 0.065 \\
neunzig_zehn & -0.469 & 0.376 & 0.641 & -0.024 & 0.227 \\
schuldenquote & 0.618 & 0.115 & -0.150 & 0.370 & 0.446 \\
beschaeftigung & 0.170 & 0.781 & 0.094 & -0.187 & 0.317 \\
bildung & 0.623 & -0.210 & 0.345 & 0.399 & 0.290 \\
lebenserw & 0.167 & -0.791 & 0.246 & -0.187 & 0.251 \\
demokratie & -0.0245 & 0.149 & -0.920 & 0.002 & 0.131 \\
treibhausg & 0.648 & 0.373 & -0.033 & -0.423 & 0.261 \\
stickstoff & 0.583 & 0.572 & 0.056 & -0.003 & 0.331 \\
vogelindex & -0.098 & 0.049 & -0.021 & 0.793 & 0.359 \\
\hline
\end{tabular}

$\mathrm{N}=14$, FA by principal-component factors with varimax. Overall Kaiser-Meyer-Olkin measure of sampling adequacy is 0,2537 (Eigene Berechnung und Darstellung).

Da jedoch keine der Variablen der $\mathrm{W}^{3}$-Indikatoren beim Kaiser-Meyer-Olkin-Kriterium den Schwellenwert von 0,5 erreicht und er für das Gesamtset nur 0,25 beträgt, ist an dieser Stelle keine Hauptkomponentenanalyse möglich. In einer alternativen Herangehensweise werden deshalb aus dem Gesamtsample drei Sub-Samples gebildet, die jeweils auf einen dahinterliegenden Faktor zurückzuführen sind. In einer iterativen Versuchsanordnung werden dafür jeweils die Gruppierungen gewählt, die die höchsten Faktorladungen produzieren. Das sind: 
Tabelle 6 Sub-Samples der Hauptkomponentenanalyse

\begin{tabular}{lllll}
\hline Sub-Sample & Indikatoren & & \\
\hline Sub-Sample 1 & $\begin{array}{l}\text { Bruttoinlandsprodukt pro } \\
\text { Beschäftigtenquote }\end{array}$ & & \\
Sub-Sample 2 & $\begin{array}{l}\text { Sekundarabschluss-II-Quote, Lebenserwartung bei Geburt, } \\
\text { Einkommensverteilung }\end{array}$ & & \\
Sub-Sample 3 & $\begin{array}{l}\text { Bruttoinlandsprodukt pro Kopf, Treibhausgas-Emissionen, } \\
\text { Stickstoffüberschuss } \\
\text { Alleinstehend }\end{array}$ & & \\
Alleinstehend & Voice and Accountability & & \\
\hline
\end{tabular}

Sub-Samnles. die ieweils gesondert einer Hauntkomnonentenanalvse unterzogen werden (eigene Darstellung).

Die beiden Leitindikatoren „Voice \& Accountability“ und „Vogelindex“ lassen sich nicht in ein Sample integrieren, da sie nicht hoch in einem der Sub-Samples laden. Darum werden sie von der Hauptkomponentenanalyse ausgeschlossen und direkt mit dem Status versehen, im Indikatorensatz unverzichtbar zu sein. Die drei SubSamples werden einer erneuten Hauptkomponentenanalyse unterzogen.

Hinter den drei Variablen des Sub-samples 1 steht ein Faktor mit einem Eigenwert größer 1, der 66,79 Prozent der Variation der Variablen erklärt. Auf alle drei Variablen lädt der Faktor bemerkenswert hoch (vgl. Tabelle A3 im Annex), die stärkste Faktorladung von $+0,87$ weist jedoch das „Bruttoinlandsprodukt pro Kopf“ auf. Das Kaiser-Meyer-Olkin-Kriterium für alle drei Variablen sowie für das gesamte Sub-Sample liegt deutlich oberhalb des Schwellenwertes 0,5; damit eignet sich das Subsample für eine Hauptkomponentenanalyse. Da es das Ziel dieser Arbeit ist, nach Reduktionsmöglichkeiten in den $\mathrm{W}^{3}$-Indikatoren zu suchen, soll im Weiteren nicht der Faktor selbst herangezogen werden, sondern diejenige Variable, die am höchsten auf den Faktor lädt, also das „Bruttoinlandsprodukt pro Kopf“ 9.

Auch im Sub-Sample 2 befindet sich hinter den drei Variablen ein gemeinsamer Faktor mit einem Eigenwert größer 1 (vgl. Tabelle A4 im Annex). Er erklärt 52,1 Prozent der Variation der drei Variablen. Wiederum liegen die kmo/KMO-Werte oberhalb der kritischen Schwelle. Am höchsten auf den Faktor laden hier die

\footnotetext{
${ }^{9}$ Die Faktorladungen können bei unabhängigen Faktoren als Korrelationskoeffizient der Variablen mit dem Faktor interpretiert werden (Eckey et al. 2002: 21).
} 
Leitindikatoren „Lebenserwartung bei Geburt" und „Einkommensverteilung“ mit quasi identischen Werten von $|0,79|$ bzw. $|0,78|$. In der Konsequenz werden beide (statt des Faktors) ins Sample übernommen.

Anhand des Sub-Samples 3 zeigt sich, dass der dahinterliegende Faktor (mit Eigenwert größer 1) 64,6 Prozent der Variation aller Variablen erklärt, dass wiederum die Variablen und das Sub-Sample selbst die kritische Kaiser-Meyer-Olkin-Schwelle überschreiten und dass es - wie bereits im Sub-Sample 1 - der Leitindikator „BIP pro Kopf“" ist, der mit 0,89 am höchsten auf den Faktor lädt (vgl. Tabelle A5 im Annex). Damit wird die Übernahme des Leitindikators in das Set der unverzichtbaren $\mathrm{W}^{3}$ Leitindikatoren aus den Ergebnissen des Sub-Samples 1 bestätigt.

Folglich kann der $\mathrm{W}^{3}$-Leitindikatorensatz auf Basis der Hauptkomponentenanalyse ohne großen Informationsverlust hinsichtlich seiner Aussagekraft in Bezug auf die gesamtgesellschaftliche Wohlfahrt auf fünf Leitindikatoren reduziert werden. Diese sind: „Bruttoinlandsprodukt pro Kopf“, „Einkommensverteilung“, „Lebenserwartung bei Geburt", "Voice and Accountability“ und "Vogelindex“. Die restlichen fünf Leitindikatoren sind über gemeinsame Faktoren so eng an die Kern-Indikatoren gebunden, dass ihre Hinzunahme in das Set keinen großen Informationsgewinn mit sich bringt.

Tabelle 7 Überblick über die Ergebnisse beider Methoden

\begin{tabular}{|l|c|c|}
\hline Leitindikator & $\begin{array}{l}\text { Ergebnisse gem. } \\
\text { Korrelationsanalyse }\end{array}$ & $\begin{array}{l}\text { Ergebnisse gem. } \\
\text { Hauptkomponentenanalyse }\end{array}$ \\
\hline BIP pro Kopf & $\mathbf{+}$ & $\mathbf{+}$ \\
\hline Einkommensverteilung & $\mathbf{+}$ & - \\
\hline Staatsschuldenquote & - & - \\
\hline Beschäftigungsquote & - & - \\
\hline $\begin{array}{l}\text { Sekundarabschluss-II- } \\
\text { Quote }\end{array}$ & $\mathbf{+}$ & $\mathbf{+}$ \\
\hline $\begin{array}{l}\text { Lebenserwartung bei } \\
\text { Geburt }\end{array}$ & - & + \\
\hline „Voice \& Accountability“ & + & - \\
\hline Treibhausgasemissionen & $\mathbf{+}$ & - \\
\hline Stickstoffüberschuss & - & $\mathbf{+}$ \\
\hline Vogelindex & $\mathbf{+}$ & \\
\hline
\end{tabular}

Die Ergebnisse beider Methoden. (+) bedeutet, dass der Indikator als Leitindikator unverzichtbar ist, (-) bedeutet, dass der Indikator entweder komplett redundant ist, oder aber als Leitindikator redundant ist und eine Klassifikation als Warnlampen-Indikator ausgereicht hätte (eigene Darstellung). 
Damit bestätigt das Ergebnis der Hauptkomponentenanalyse das Ergebnis der Korrelationsanalyse in weiten Teilen. Beide Verfahren legen nahe, dass das „Bruttoinlandsprodukt pro Kopf", die „Einkommensverteilung“, „Voice \& Accountability" sowie der "Vogelindex" als Leitindikatoren unverzichtbar sind. Während die Korrelationsanalyse jedoch auch die Beibehaltung der Leitindikatoren „Sekundarabschluss-II-Quote" sowie „Treibhausgasemissionen“ nahelegt, ist gemäß Hauptkomponentenanalyse zusätzlich lediglich die „Lebenserwartung bei Geburt" unverzichtbar. Aus der Betrachtung der Wohlfahrt herausgenommen werden können damit laut beiden Methoden die Leitindikatoren "Staatsschuldenquote“, „Beschäftigungsquote“ sowie „Stickstoffüberschuss“. Die zehn W'3-Leitindikatoren ließen sich somit auf sieben reduzieren, ohne größere Informationsverluste über die Entwicklung der Wohlfahrt in Deutschland hinnehmen zu müssen. Legt man das etwas schwächere Kriterium an, dass ein Leitindikator bereits verzichtbar ist, wenn er nur von einer der beiden Methoden als überflüssig eingestuft wurde, so ließe sich der Indikatorensatz auf die vier von beiden Methoden als unverzichtbar erwiesenen Leitindikatoren reduzieren.

\section{Fazit: $\mathbf{W}^{3}$-Leitindikatoren reduzieren}

Alternative Ansätze zur Messung von Wohlfahrt haben in den vergangenen Jahren an Bedeutung gewonnen. Eine Möglichkeit ist die Bildung von Indikatorensätzen, bei deren Konstruktion die zentrale Frage lautet: Wie viele Einzelindikatoren können hinzugenommen werden, bevor der Satz zu umfangreich und komplex wird und damit unweigerlich an öffentlicher Relevanz verliert?

Im Frühjahr 2013 hat die Enquete-Kommission „Wachstum, Wohlstand, Lebensqualität - Wege zu nachhaltigem Wirtschaften und gesellschaftlichem Fortschritt in der Sozialen Marktwirtschaft" mit den $\mathrm{W}^{3}$-Indikatoren einen alternativen Wohlfahrtsindikatorensatz vorgelegt, mit dem grundsätzliche Defizite des Bruttoinlandsprodukts in seiner Aussagekraft zur gesamtgesellschaftlichen Wohlfahrt behoben werden sollen. Damit ist die ohnehin schon große Familie alternativer Ansätze zur Wohlfahrtsmessung um einen weiteren Ansatz reicher.

Mit Hilfe von Korrelations- und Hauptkomponentenanalysen wurde auf Basis nationaler Zeitreihen und internationaler Querschnittsdaten untersucht, ob sich Redundanzen in den Leitindikatoren der $\mathrm{W}^{3}$-Indikatoren nachweisen lassen. Die statistische Auswertung durch beide Methoden zeigt, dass bei den zehn 
Leitindikatoren die Möglichkeit bestanden hätte, zumindest auf drei Indikatoren zu verzichten. Beide Methoden liefern Hinweise dafür, dass die Leitindikatoren "Staatsschuldenquote", „Beschäftigungsquote“ sowie "Stickstoffüberschuss" redundant sind. Das bedeutet: Mit ihrer Aufnahme in den Satz der W' ${ }^{3}$ Indikatoren geht kein großer Informationsgewinn in Fragen der Wohlfahrt einher, da sie zu einem guten Teil von den restlichen $W^{3}$-Indikatoren erklärt werden. Unverzichtbar als $\mathrm{W}^{3}$-Leitindikatoren sind damit "Bruttoinlandsprodukt pro Kopf“, „Einkommensverteilung", „Sekundarabschluss-II-Quote“, „Lebenserwartung bei Geburt", „Voice \& Accountability“, "Treibhausgasemissionen“ sowie der "Vogelindex“. Legt man das etwas schwächere Kriterium an, dass ein Leitindikator bereits wegfallen kann, sobald er nur von einer der beiden Methoden als verzichtbar eingestuft wird, so ließe sich der Satz an Leitindikatoren sogar auf nur vier Stück verdichten - nämlich diejnigen, die von beiden Methoden als unverzichtbar eingestuft wurden. Dabei handelt es sich um "Bruttoinlandsprodukt pro Kopf“, „Einkommensverteilung", „Voice and Accountability“ und den „Vogelindex“. Ob bei der Reduktion des Indikatorensatzes ein mehr oder weniger strenges Kriterium angelegt wird - ob er angesichts der Ergebnisse dieser Analyse also auf sieben oder vier Leitindikatoren reduziert wird - bleibt eine politische Entscheidung.

Die Ergebnisse untermauern die Bedeutung, die einer kritischen begleitenden statistischen Analyse bei der Erstellung von Wohlfahrtsmaßen zukommt. Interessant wäre zudem, ob sich die hiesigen Ergebnisse auch an anderen bekannten und auch national wie international angewandten Indikatorensätzen (bspw. der „How is Life?“Indikatorensatz der OECD oder der Indikatorensatz der Nachhaltigkeitsstrategie der Bundesregierung) mit den hier angewandten Methoden bestätigen ließen.

\section{Literaturverzeichnis}

Acemoglu, Daron; Robinson, James A. (2006): Economic origins of dictatorship and democracy. Cambridge, New York: Cambridge University Press.

Agnello, Luca; Sousa, Ricardo M. (2012): Fiscal Adjustments and Income Inequality - A First Assessment. Nucleo de Investigacao em Politicas Economicas - Universidad Minde Minho. Minho (NIPE-Working-Paper, 19). Online verfügbar unter http://ideas.repec.org/p/nip/nipewp/19-2012.html, zuletzt geprüft am 02.01.2014.

Azzimonti, Marina; Di Francisco, Eva; Quadrini, Vincenzo (2012): Financial Globalization, Inequality, and the Rising of Public Debt. Hg. v. F. PhiladelphiaE.D. Research Department. FED Philadelphia. Philadelphia (CEPR-Diskussio-Paper, 126). Online verfügbar unter http://ideas.repec.org/p/cpr/ceprdp/8893.html, zuletzt geprüft am 02.01.2014. 
Baek, Jungho; Gweisah, Guankerwon (2013): Does income inequality harm the environment?: Empirical evidence from the United States. In: Energy Policy 62, S. 1434-1437.

Banerjee, Abhijit V.; Duflo, Esther (2003): Equality and Growth: What can the data say. In: Journal of Economic Growth 8, S. 267-299.

Bardt, Hubertus (2012): Mehr Wohlstand mit weniger Umweltverbrauch. In: Institut der Deutschen Wirtschaft (IW) (Hg.): Wirtschaftswachstum?! Warum wir wachsen sollten und warum wir wachsen können. Köln: IW Medien GmbH (IW-Studien), S. 95-109.

Bardt, Hubertus; Grömling, Michael; Kroker, Rolf (2012): Führt Wachstum zu mehr Wohlstand? In: Institut der Deutschen Wirtschaft (IW) (Hg.): Wirtschaftswachstum?! Warum wir wachsen sollten und warum wir wachsen können. Köln: IW Medien $\mathrm{GmbH}$ (IW-Studien), S. 47-72.

Barro, Robert J. (2000): Inequality and Growth in a Panel of Countries. In: Journal of Economic Growth 5 (1), S. 5-32.

Becketti, Sean (2013): Introduction to Time Series Using Stata. College Station, Texas: Stata Press.

Bengochea-Morancho, Aurella; Higon-Tamarit, Francisco; Martinez-Zarzoso, Immaculada (2001): Economic Growth and CO2-Emissions in the European Union. In: Environmental and Resource Economics 19, S. 165-172.

Besley, Timothy; Kudamatsu, Masayuki (2006): Health and Democracy. In: American Economic Review 96 (2), S. 313-318.

Biewen, Martin; Juhasz, Andos (2010): Understanding Rising Inequality in Germany. Forschungsinstitut für die Zukunft der Arbeit (IZA). Bonn (IZA Discussion-Papers, 5062). Online verfügbar unter http://www.Isw.wiso.unierlangen.de/VfS/Vortr_ge/Biewen_dp5062.pdf., zuletzt geprüft am 02.01.2014.

Blanchard, Olivier Jean; Fischer, Stanley (1993): Lectures on Macroeconomics. 6. Aufl. Cambridge, Massachusetts: The MIT Press.

Blanden, J.; Machin, S. (2010): Education and Inequality. In: Dominic J. Brewer und Patrick J. McEwan (Hg.): Economics of education. Oxford, UK, San Diego, CA: Acadmic Press, S. 99-108.

BMELV (Bundesministerium für Ernährung, Landwirtschaft und Verbraucherschutz) (2016): Nährstoffbilanz insgesamt von 1990 bis 2011 - in kg N/ha. Berlin. Online verfügbar unter http://berichte.bmelv-statistik.de/MBT-0111260-0000.xls; zuletzt geprüft am 03.07.2016.

Boarini, Romina; Johansson, Asa; Ercole, Marco Mira (2006): Alternative Measures of Well-Being. OECD. Paris (OECD Social, Employment and Migration Working Papers, 33). Online verfügbar unter http://www.oecd.org/els/soc/36165332.pdf, zuletzt geprüft am 02.10.2013.

Brügelmann, Ralph (2012): Wachstum und öffentliche Haushalte. In: Institut der Deutschen Wirtschaft (IW) (Hg.): Wirtschaftswachstum?! Warum wir wachsen sollten und warum wir wachsen können. Köln: IW Medien GmbH (IW-Studien), S. 111-130.

Bundesamt für Naturschutz (BfN) (2013): Indikator "Artenvielfalt und Landschaftsqualität". Bundesamt für Naturschutz (BfN). Berlin. 
Cantarero, David; Pascual, Marta; María Sarabia, José (2005): Effects of income inequality on population health: new evidence from the european community household panel. In: Applied Economics 37 (1), S. 87-91.

Campbell, Kathryn; Cooper, David; Dias, Braulio; Prieur-Richard, Anne-Hélène; Campbell-Lendrum, Diarmid; Karesh, William B.; Daszak, Peter (2011): Strengthening International Cooperation for Health and Biodiversity. In: EcoHealth 8 (4), S. 407-409.

Checherita-Westphal, Cristina; Rother, Philipp (2012): The impact of high government debt on economic growth and its channels: An empirical investigation for the euro area. In: European Economic Review 56 (7), S. 1392-1405.

Coccia, Mario (2012): What are the effects of public debt on innovation and employment growth? National Research Council of Italy (CNR), ISTITUTO DI RICERCA SULL'IMPRESA E LO SVILUPPO (Ceris). Moncalieri (CNR-Ceris Working Paper, 06/2012). Online verfügbar unter http://econpapers.repec.org/paper/csccerisp/201206.htm, zuletzt geprüft am 05.01.2014.

Dee, Thomas S. (2004): Are there civic returns to education? In: Journal of Public Economics 88 (9-10), S. 1697-1720.

Diefenbacher, Hans (2009): Indikatoren nachhaltiger Entwicklung für die Bundesrepublik Deutschland. Zwischenbilanz einer Wanderung zwischen Theorie und Praxis. In: Reinhold Popp (Hg.): Zukunftsforschung und Zukunftsgestaltung. Beiträge aus Wissenschaft und Praxis. Berlin: Springer (Wissenschaftliche Schriftenreihe Zukunft und Forschung des Zentrums für Zukunftsstudien Salzburg, 1), S. 684-694.

Diefenbacher, Hans (2013): Nationaler Wohlfahrtsindex: Pro Echtes Wohlfahrtsmaß. In: Wirtschaftsdienst - Zeitschrift für Wirtschaftspolitik 93 (2), S. 66-67.

Dietz, Dimon; Adger, W. Neil (2003): Economic Growth, biodiversity loss and conservation effort. In: Journal of Environmental Management 68 (1), S. 23-35.

Dise, Nancy B. (2011): Chapter 20: Nitrogen as a threat to European terrestrial biodiversity. In: The European nitrogen assessment. Sources, effects, and policy perspectives. Cambridge, UK, New York: Cambridge University Press, S. 463-494.

Deutsche Bundesbank (2013): Zeitreihe BBK01.BJ9959. Verschuldung gem. Maastricht-Vertrag - Deutschland - Gesamtstaat - in \% des BIP. Frankfurt a. Main. Online verfügbar

unter http://www.bundesbank.de/Navigation/DE/Statistiken/Zeitreihen_Datenbanken/Makro oekonomische_Zeitreihen/its_details_value_node.html?listld=www_v27_web001_02 a\&tsld=BBK01.BJ9959, zuletzt aktualisiert am 10.10.2013, zuletzt geprüft am 15.10.2013.

DIW (2013): SOEP 1011 - SOEPmonitor Person 1984-2011. (SOEP v28). Deutsches Institut für Wirtschaftsforschung (DIW). Berlin (SOEP Survey Papers - Series E 119). Online verfügbar unter http://www.diw.de/de/diw_02.c.222728.de/soepmonitor.html, zuletzt geprüft am 08.02.2014.

Eckey, Hans-Friedrich; Kosfeld, Reinhold; Rengers, Martina (2002): Multivariate Statistik. Grundlagen - Methoden - Beispiele. 1. Aufl. Wiesbaden: Gabler (Lehrbuch). 
Eide, E. R.; Showalter, M. H. (2010): Human Capital. In: Dominic J. Brewer und Patrick J. McEwan (Hg.): Economics of education. Oxford, UK, San Diego, CA: Acadmic Press, S. 27-32.

Enquete-Kommission "Wachstum, Wohlstand Lebensqualität" (2013): Schlussbericht. Hg. v. Deutscher Bundestag. Berlin (Bundestagsdrucksache, 17/13300).

Eurostat (2013a): Zeitreihe: edat_Ifse_08. Persons with upper secondary or tertiary education attainment by age and sex (\%). Hg. v. Eurostat Database. Luxemburg. Online verfügbar unter http://appsso.eurostat.ec.europa.eu/nui/show.do?dataset=edat_Ifse_08\&lang=en, zuletzt aktualisiert am 10.09.2013, zuletzt geprüft am 15.10.2013.

Eurostat (2013b): Zeitreihe NAMA_AUX_GPH: Bruttoinlandsprodukt pro Kopf - jährliche Daten (hier reales BIP). Luxemburg. Online verfügbar unter http://epp.eurostat.ec.europa.eu/portal/page/portal/product_details/dataset?p_produc t_code=NAMA_AUX_GPH, zuletzt aktualisiert am 15.10.2013, zuletzt geprüft am 23.10.2013.

Eurostat (2013d): Zeitreihe GOV_DD_EDPT1: Defizit/Überschuss, Schuldenstand des Staates und damit zusammenhängende Daten. Online verfügbar unter http://epp.eurostat.ec.

europa.eu/portal/page/portal/product_details/dataset?p_product_code=GOV_DD_ED PT1, zuletzt aktualisiert am 21.10.2013, zuletzt geprüft am 23.10.2013.

Eurostat (2013e): Zeitreihe LFSA_ERGAN: Erwerbstätigenquote nach Geschlecht, Alter und Staatsangehörigkeit ${ }^{-}$(\%). Online verfügbar unter http://epp.eurostat.ec.europa.eu/portal /page/portal/product_details/dataset?p_product_code=LFSA_ERGAN, zuletzt aktualisiert am 15.10.2013, zuletzt geprüft am $2 \overline{3} .10 .2013$.

Eurostat (2013f): Datensatz ENV_AIR_GGE: Treibhausgasemissionen (Quelle: EUA). Luxemburg. Online $\quad$ verfügbar unter http://epp.eurostat.ec.europa.eu/portal/page/portal/product details/dataset?p_product_code=ENV_AIR_GGE, zuletzt aktualisiert am 18.10.2013, zuletzt geprüft am 23.10.2013.

Eurostat (2013g): Zeitreihe AEI_PR_GNB: Bruttonährstoffbilanz. Online verfügbar unter http://epp.eurostat.ec.europa.eu/portal/page/portal/product_details/dataset?p_produc t_code=AEI_PR_GNB, zuletzt aktualisiert am 09.01.2013, zuletzt geprüft am 23.10.2013.

Eurostat (2013h): Zeitreihe ENV_BIO2: Schutz der Natürlichen Ressourcen - Index weit verbreiteter Vogelarten. Luxemburg. Online verfügbar unter http://epp.eurostat.ec.europa.eu/portal/page/portal/product_details/dataset?p_produc $\mathrm{t}$ code=ENV_BIO2, zuletzt aktualisiert am 25.09.2012, zuletzt geprüft am 23.10.2013.

Eurostat (2013i): Distribution of income by quantiles. Zeitreihe ilc_di01. Eurostat. Luxemburg. Online unter: http://appsso.eurostat.ec.europa.eu/nui/show.do?dataset=ilc_di01\&lang=en, zuletzt geprüft am 08.02.2014.

Eurostat (2013j): Bevölkerung am 1. Januar. Eurostat. Luxemburg. Online verfügbar unter

http://epp.eurostat.ec.europa.eu/tgm/table.do?tab=table\&language=de\&pcode=tps00 
001\&tableSelection=1\&footnotes=yes\&labeling=labels\&plugin=1, zuletzt geprüft am 08.02.2014.

Farzanegan, Mohammad Reza; Markwardt, Gunther (2012): Pollution, Economic Development and Democracy: Evidence from the MENA countries. Hg. v. Universität Marburg. Marburg (Jount Discussion Paper Series i Economics by the Universities of Aachen, Gießen, Göttingen, Kasel, Marburg, Siegen, 27-2012).

Földvári, Péter; van Leeuwen, Bas (2011): Should less inequality in education lead to more equal income distribution? In: Education Economics 19 (5), S. 537-554.

Franco, Alvaro; Alvarez-Dardet, Carlos; Ruiz, Maria Teresa (2004): Effect of Democracy on Health: Ecological Study. In: British Medical Journal 329, S. 1421-1424.

Galor, Oded (2011): Inequality, Human Capital Formation, and the Process of Development. In: Eric Alan Hanushek, Stephen Machin und Ludger Woessmann (Hg.): Handbook of the Economics of Education. 4. Aufl. Amsterdam: North-Holland, S. 442-493.

Glaeser, Edward L.; Ponzetto, Giacomo A. M.; Shleifer, Andrei (2007): Why does democracy need education? In: Journal of Economic Growth 12 (2), S. 77-99.

Gregorio, José de; Lee, Jong-Wha (2002): Education and Income Inequality: New Evidence from Cross-Country Data. In: Review of Income and Wealth 48 (3), S. 395416.

Grömling, Michael; Schröder, Christop (2012): Erhöht Wachstum die Ungleichheit? In: Institut der Deutschen Wirtschaft (IW) (Hg.): Wirtschaftswachstum?! Warum wir wachsen sollten und warum wir wachsen können. Köln: IW Medien GmbH (IWStudien), S. 149-171.

Grunwald, Armin; Kopfmüller, Jürgen (2012): Nachhaltigkeit. 2. Aufl. Frankfurt am Main: Campus (Reihe Campus Studium).

Haines, Andy (2012): Sustainable policies to improve health and prevent climate change. In: Social Science \& Medicine 74 (5), S. 680-683.

Hanushek, Eric A.; Woessmann, Ludger (2010): Education and Economic Growth. In: Dominic J. Brewer und Patrick J. McEwan (Hg.): Economics of education. Oxford: Elsevier; Academic Press.

Hazan, Moshe (2012): Life expectancy and schooling: new insights from cross-country data. In: Journal of Population Economics 25 (4), S. 1237-1248.

Heerink, Nico; Mulatu, Abay; Bulte, Erwein (2001): Income Inequality and the environment: aggregation bias in environmental Kuznets curve. In: Ecological Economics 38 (3), S. 359-367.

Hérault, Nicolas; Kostenko, Weiping; Marks, Gary; Zakirova, Rezida (2012): The Effects of Macroeconomic Conditions on the Education and Employment Outcomes of Youth. In: Australian Journal of Labour Economics 15 (1), S. 17-36.

Herndon, Thomas; Ash, Michael; Pollin, Robert (2010): Does High Public Debt Consistently Stifle Economic Growth? A Critique of Reinhard and Rogoff. Political Economy Research Institute, University of Massachusetts Amherst. Amherst, Massachusetts (Working Paper Series, 322). Online verfügbar unter http://www.peri.umass.edu/236/hash/31e2ff374b6377b2ddec04deaa

6388b1/publication/566/, zuletzt geprüft am 30.12.2013. 
Holland, T. Gi.M.; Peterson, Garry D.; Gonzalez, Andrew (2009): A Cross-National Analysis of How Economic Inequality Predicts Biodiversity Loss. In: Conservation Biology 23 (5), S. 1304-1313.

Houle, Christian (2009): Inequality and Democracy: Why Inequality Harms Consolidation but does not affect Democratization. In: World Politics 61 (4), S. 589622.

IPCC (2007): Fourth Assessment Report - Chapter 4: Ecosystems, their properties, goods and services. Intergovernmental Panel on Climate Change (IPCC). Cambridge. Online verfügbar unter http://www.ipcc.ch/publications_and_data/ar4/wg2/en/ch4.html, zuletzt geprüft am 11.01.2014.

Jorgenson, Andrew K. (2003): Consumption and Environmental Degradation: A CrossNational Analysis of the Ecological Footprint. In: Social Problems 50 (3), S. 374-394.

Kaether, Johann; Stöckmann, Maik; Behrens, Hermann (2005): Freiwillige Selbstkontrolle Nachhaltigkeit (FSK-N) in Region und Stadt. Akteursorientierte Entwicklung und Erprobung eines regionalen Berichtssystems für eine nachhaltige Regionalentwicklung am Beispiel der Region Mecklenburgische Seenplatte. Hochschule Neubrandenburg. Neubrandenburg. Online verfügbar unter www.ssv.hsnb.de/fileadmin/IUGR/fskn.pdf. Zuletzt geprüft am 17.02.2014.

Kassenböhmer, Sonja C.; Schmidt, Christoph M. (2011): Beyond GDP and Back: What is the Value-Added by Additional Components of Welfare Measurement? Deutsches Institut für Wirtschaftsforschung (DIW). Berlin (SOEPpapers). Online verfügbar unter: http://www.diw.de/sixcms/detail.php?id=diw_01.c.368544.de. Zuletzt geprüft am 18.02.2014.

Kennedy, Ryan (2010): The Contradiction of Modernization: A Conditional Model of Endogenous Democratization. In: Journal of Politics 72 (03), S. 785-798.

Kibele, E. U. B.; Jasilionis, D.; Shkolnikov, V. M. (2013): Widening socioeconomic differences in mortality among men aged 65 years and older in Germany. In: Journal of Epidemiology \& Community Health 67 (5), S. 453-457.

Kinda, Somlanaré Romuald (2010): Does Education really Matter for Environmental Quality? Hg. v. Université d'Auvergne. Centre d'études et de recherche sur le dévelopement international (CERDI). Clermont Ferrand (Etudes et Documents, 2010.28). Online verfügbar unter http://halshs.archivesouvertes.fr/docs/00/55/22/25/PDF/2010.28.pdf.

Kumar, Manmohan S.; Woo, Jaejoon (2010): Public Debt and Growth. International Monetary Fund. Washington (IMF Working-Paper-Series, 10/174). Online verfügbar unter https://www.imf.org/external/pubs/ft/wp/2010/wp10174.pdf, zuletzt geprüft am 30.12.2013.

Kuznets, Simon (1955): Economic Growth and Income Inequality. In: American Economic Review 45 (1), S. 1-28.

Lora, Eduardo; Olivera, Mauricio (2007): Public Debt and Social Expenditure: Friends or Foes? In: Emerging Markets Review 8 (4), S. 299-310.

Machin, S.; van Reenen, John (2008): Changes in Wage Inequality. In: Steven N. Durlauf und Lawrence E. Blume (Hg.): The new Palgrave dictionary of economics. 2. Aufl. Houndmills, Basingstoke [u.a.]: Palgrave Macmillan, S. 659-666.

Mankiw, N. Gregory (2010): Macroeconomics. 7. ed., 2. print. New York, NY: Worth. 
Mattoscio, Nicola; Bucciarelli, Edgardo; Odoardi, lacopo; Ernesto, Persico Toni (2012): An Examination of Okun's Law: Evidence from European Target Countries. In: Annals of Faculty of Economics, University of Oradea 1 (2), S. 237-243.

McMahon, W. W. (2010): The External Benefits of Education. In: Dominic J. Brewer und Patrick J. McEwan (Hg.): Economics of education. Oxford, UK, San Diego, CA: Acadmic Press, S. 68-79.

McMichael, A. J.; Lindgren, E. (2011): Climate change: present and future risks to health, and necessary responses. In: Journal of Internal Medicine 270 (5), S. 401413.

McMichael, Anthony (2013): Globalization, Climate-Change, and Human Health. In: The New England Journal of Medicine 368 (14), S. 1335-1343.

Muening, P. (2010): Education and Health. In: Dominic J. Brewer und Patrick J. McEwan (Hg.): Economics of education. Oxford, UK, San Diego, CA: Acadmic Press, S. 80-87.

Nachhaltigkeitsrat (2010): Nachhaltigkeits-Indikatoren zur Messung der gesamtwirtschaftlichen Entwicklung. Gutachten an den Sachverständigenrat zur Begutachtung der gesamtwirtschaftlichen Entwicklung zum Bericht der Stiglitz-SenFitoussi-Kommission. Hg. v. Geschäftsstelle des Rates für Nachhaltige Entwicklung. Rat für Nachhaltige Ontwicklung. http://www.nachhaltigkeitsrat.de/uploads/media/RNE_Gutachten_zum_Bericht_der_S tiglitz-Sen-Fitoussi-Kommission_31-05-2010_01.pdf, zuletzt geprüft am 08.01.2013.

Obinger, Herbert (2004): Politik und Wirtschaftswachstum. Ein internationaler Vergleich. 1 Band. Wiesbaden: VS Verlag für Sozialwissenschaften.

OECD (2008): Handbook on constructing composite indicators. Methodology and user guide. Paris: OECD.

OECD (2013a): Zeitreihe: Health Status - Life Expectancy - Total Population at Birth. Hg. v. OECD Database. Paris. Online verfügbar unter http://stats.oecd.org/Index.aspx? DatasetCode =HEALTH_STAT, zuletzt aktualisiert am Oktober 2013, zuletzt geprüft am 23.10.2013.

OECD (2013b): Education at a glance. How does educational attainment affect participation in the labour market? Hg. v. OECD. Paris. Online verfügbar unter http://www.keepeek.com/Digital-Asset-Management/oecd/education/education-at-aglance-2013/indicator-a5-how-does-educational-attainment-affect-participation-in-thelabour-market_eag-2013-9-en\#page2, zuletzt geprüft am 25.01.2014.

Okun, Arthur M. (1962): Potential GNP: Its Measurement and Significance. Proceedings of the Business and Economics Statistics Section of the American Statistical Association, S. 98-104.

Perotti, Roberto (1996): Growth, Income Distribution, and Democracy: What the Data Say. In: Journal of Economic Growth 1 (2), S. 149-187.

Piaggio, Matias; Padilla, Emilio (2012): CO2-Emissions and Economic Growth: Heterogeneity Across Countries and Non-Stationary Series. In: EnergyPolicy 46 (C), S. 370-381. 
Reinhard, Carmen M.; Rogoff, Kenneth S. (2010): Growth in a Time of Debt. In: American Economic Review 100 (2), S. 573-578.

Riddell, W. Craig; Song, Xueda (2004): The impact of education on unemployment incidence and re-employment-success: Evidence from the US-labour-market. Forschungsinstitut zur Zukunft der Arbeit (IZA). Bonn (IZA Discussion-Papers, 5572). Online verfügbar unter https://www.econbiz.de/Record/the-impact-of-education-onunemployment-incidence-and-re-employment-success-evidence-from-the-us-labourmarket-riddell-craig/10009233917, zuletzt geprüft am 07.01.2014.

Rodríguez-Pose, Andrés; Tselios, Vassilis (2009): Education and Income Inequality in the Regions of the European Union. In: Journal of Regional Science 49 (3), S. 411437.

Salti, Nisreen (2010): Income Inequality and the Composition of Public Debt. American University of Beirut - Department of Economics. Beirut. Online verfügbar unter www.erf.org.eg /CMS/getFile.php?id=1851, zuletzt geprüft am 02.01.2014.

Schmidt, Christoph M.; Aus dem Moore, Nils (2012): Lebensqualität - Wie lässt sich Wohlstand messen? In: Ulrich van Suntum (Hg.): Die Wohlstandsfrage. 6 Visionen für nachhaltiges Wachstum. Berlin: Initiative Neue Soziale Marktwirtschaft - INSM $\mathrm{GmbH}$, S. 57-81.

Schmid, Daniel Kai; Stein, Ulrike (2013): Explaining Rising Income Inequality in Germany 1991 - 2010. Institut für Makroökonomie und Konjunkturforschung (IMK). Düsseldorf (IMK-Study, 32). Online verfügbar unter www.boeckler.de/pdf/p_imk_study_32_2013.pdf, zuletzt geprüft am 02.01.2014.

Schneider, Brit S. (2007): Gesundheit und Bildung. Theorie und Empirie der Humankapitalinvestitionen. Frankfurt am Main [u.a.]: Lang (Allokation im marktwirtschaftlichen System, 54).

Sianesi, Barbara; Van Reenen, John (2003): The Returns to Education: Macroeconomics. In: Journal of Economic Surveys 17 (2), S. 157-200.

Sirowy, Larry; Inkeles, Alex (1990): The Effects of Democracy on Economic Growth and Inequality: A Review. In: Studies in Comparative International Development 25 (1), S. 126-157.

Stata (2005): Stata Time-Series - Reference Manual Release 9. College Station, Texas: StataCorp LP.

Statistisches Bundesamt (2013a): Volkswirtschaftliche Gesamtrechnungen. Bruttoinlandsprodukt, Bruttonationaleinkommen, Volkseinkommen - Lange Reihen ab 1950. Wiesbaden.

Online: https://www.destatis.de/DE/ZahlenFakten/GesamtwirtschaftUmwelt/VGR/Inlandsprod ukt/Tabellen/Volkseinkommen1950.xlsx?__blob=publicationFile, zuletzt geprüft am 15.10.2013.

Statistisches Bundesamt (2013b): Bevölkerung und Erwerbstätigkeit. Vorläufige Ergebnisse der Bevölkerungsfortschreibung auf Grundlage des Zensus 2011. Statistisches Bundesamt. Online: https://www.destatis.de/DE/Publikationen/Thematisch/Bevoelkerung/Bevoelkerungsst and/VorlBevoelkerungsfortschreibung.html, zuletzt geprüft am 15.10.2013.

Statistisches Bundesamt (2013c): Zeitreihe 12211-0001. Bevölkerung, Erwerbstätige, Erwerbslose, Erwerbspersonen, Nichterwerbspersonen [jeweils im Alter von 15 bis unter 65 Jahren]: Deutschland, Jahre, Geschlecht. Hg. v. Destatis/Genesis- 
Datenbank. Wiesbaden. Online verfügbar unter https://wwwgenesis.destatis.de/genesis/online/link/tabelleErgebnis/12211-0001, zuletzt aktualisiert am 15.10.2013, zuletzt geprüft am 15.10.2013.

Statistisches Bundesamt (2012): Nachhaltige Entwicklung in Deutschland, Indikatorenbericht 2012. Hg. v. Statistisches Bundesamt. Wiesbaden. Online verfügbar unter https://www.destatis.de/DE /Publikationen/Thematisch/UmweltoekonomischeGesamtrechnungen/Umweltindikato ren/IndikatorenPDF_0230001.html, zuletzt geprüft am 04.02.2013.

Stern, David I. (2004): The Rise and Fall of the Environmental Kuznets Curve. In: World Development 32 (8), S. 1419-1439.

Stevens, Carly J.; Dise, Nancy B.; Mountford, J. Owen; Gowing, David J. (2004): Impact of Nitrogen Deposition on the Species Richness of Grasslands. In: Science 303, S. 1876-1879.

Stiglitz, Joseph; Sen, Amartya; Fitoussi, Jean-Paul (2009): Report by the Comission on the Measuremt of Performance and Social Progress. Hg. v. French Government. Online verfügbar unter http://www.stiglitz-sen-fitoussi.fr/en/index.htm, zuletzt geprüft am 12.01.2014.

SVR (Sachverständigenrat zur Begutachtung der gesamtwirtschaftlichen Entwicklung) (2010): Wirtschaftsleistung, Lebensqualität und Nachhaltigkeit. Ein umfassendes Indikatorensystem ; Expertise im Auftrag des Deutsch-Französischen Ministerrates. Wiesbaden. Online verfügbar unter http://www.sachverstaendigenratwirtschaft.de/expertisen.html?\&no_cache=1, zuletzt geprüft am 17.02.2014.

Szabó, Zsolt (2013): The Effect of Sovereign Debt on Economic Growth and Economic Development. In: State Audit Office of Hungary, Public Finance Quaterly 58 (3), S. 251-270.

Thuiller, Wilfried (2007): Climate Cahnge and the Ecologist. In: Nature 448, S. 550-552.

Torras, Mariano; Boyce, James K. (1998): Income Inequality, and pollution: a reassessment of the environmental Kuznets curve. In: Ecological Economics 25 (2), S. $147-160$.

Van Suntum, Ulrich; Lerbs, Oliver (2011): Theoretische Fundierung und Bewertung alternativer Methoden der Wohlfahrtsmessung, Studie im Auftrag der KfWBankengruppe, Münster. Online verfügbar unter: https://www.kfw.de/DownloadCenter/Konzernthemen/Research/PDF-Dokumente-Sonderpublikationen/AlternativeMethoden-der-Wohlfahrtsmessung.pdf, zuletzt geprüft am 14.02.2014.

Voß, Werner (2004): Taschenbuch der Statistik. 2. Aufl. Leipzig: Fachbuchverlag Leipzig.

Weltbank (2013): Worldwide Governance Indicators. Voice and Accountability, Percentlie Rank, Germany. Hg. v. Worldbank Database. Washington. Online verfügbar unter http://databank.worldbank.org/data/views/variableselection/selectvariables.aspx?sour ce=worldwide-governance-indicators\#, zuletzt geprüft am 15.10.2013.

Wilkinson, Richard G.; Pickett, Kate (2010): Gleichheit ist Glück. Warum gerechte Gesellschaften für alle besser sind. 3. Aufl. Berlin: Tolkemitt bei Zweitausendeins.

Yi, Dae Jin (2012): No taxation, no democracy? Taxation, income inequality and democracy. In: Journal of Economic Policy Reform 15 (2), S. 71-92. 
ANNEX

Tabelle A 1: Datenstruktur der Längsschnittdaten (Deutschland)

\begin{tabular}{|l|lllll|}
\hline Variable & Obs & Mean & Std. Dev. & Min & Max \\
\hline bip_real_pc & 22 & 26434.18 & 2332.413 & 23234.5 & 30695.97 \\
neunzig_zehn & 22 & 3.087727 & .1625666 & 2.88 & 3.37 \\
schuldenquote & 22 & 62.20455 & 11.58881 & 39.5 & 82.4 \\
beschaeftigung & 23 & 66.73391 & 2.772934 & 63.69 & 72.63 \\
bildung & 20 & 75.35 & 3.401316 & 71.4 & 82.8 \\
\hline lebenserw & 22 & 78.15455 & 1.726192 & 75.3 & 80.8 \\
demokratie & 14 & 92.65 & 1.681199 & 88.94 & 94.71 \\
treibhausg & 23 & 1051627 & 91592.36 & 911578 & 1250518 \\
stickstoff & 22 & 110.6018 & 11.96164 & 83.79 & 147.81 \\
vogelindex & 21 & 70.08571 & 3.519416 & 62.7 & 75.7 \\
\hline
\end{tabular}

Datenstruktur des Zeitreihendatensatzes für Deutschland. Betrachtete Periode: maximal 1990 - 2012 (eigene Berechnung und Darstellung).

Tabelle A 2: Datenstruktur der Querschnittsdaten (EU-28)

\begin{tabular}{|l|lllll|}
\hline Variable & Obs & Mean & Std. Dev. & Min & Max \\
\hline bip_real_pc & 28 & 21782.14 & 14613.62 & 3700 & 68700 \\
neunzig_zehn & 27 & 3.754444 & .8758791 & 2.77 & 5.76 \\
schuldenquote & 27 & 47.01481 & 28.43406 & 4.5 & 112.9 \\
beschaeftigung & 28 & 66.02143 & 6.037313 & 55.3 & 77.9 \\
bildung & 28 & 68.47857 & 13.86745 & 28.4 & 84.2 \\
\hline lebenserw & 21 & 79.07619 & 2.399147 & 74.1 & 81.8 \\
demokratie & 26 & 85.11423 & 11.49212 & 59.13 & 100 \\
treibhausg & 28 & 177984.9 & 237142.6 & 3061 & 974993 \\
stickstoff & 27 & 57.81481 & 45.72496 & -18 & 188 \\
vogelindex & 15 & 92.18 & 11.54978 & 75.7 & 115.2 \\
\hline
\end{tabular}

Datenstruktur der Querschnittsdaten für die EU-28. Betrachtetes Jahr: 2008 (eigene Berechnung und Darstellung) 
Tabelle A3: Ergebnisse der Hauptkomponentenanalyse am Sub-Sample 1

\begin{tabular}{|l|rrrr|}
\hline Factor & Eigenvalue & Difference & Proportion & Cumulative \\
\hline Factor1 & 2.004 & 1.410 & 0.668 & 0.668 \\
Factor2 & 0.594 & 0.192 & 0.198 & 0.866 \\
Factor3 & 0.402 &. & 0.134 & 1.000 \\
\hline
\end{tabular}

\begin{tabular}{|l|l|l|l|r|}
\hline Factor & Factor1 & Uniqueness & Variable & kmo \\
\hline bip_real_pc & 0.865 & 0.252 & bip_real_pc & 0.624 \\
schuldenquote & -0.791 & 0.374 & schuldenquote & 0.697 \\
beschaeftigung & 0.794 & 0.370 & beschaeftigung & 0.694 \\
\hline & & & Overall & 0.665 \\
\hline
\end{tabular}

Ergebnisse für das Sub-Sample 1: N=21, FA by principal component factors with varimax. Overall Kaiser Meyer-Olkin measure of sample adequacy is 0,67 (eigene Berechnung und Darstellung).

Tabelle A4: Ergebnisse der Hauptkomponentenanalyse am Sub-Sample 2

\begin{tabular}{|l|rrrr|}
\hline Factor & Eigenvalue & Difference & Proportion & Cumulative \\
\hline Factor1 & 1.562 & 0.710 & 0.521 & 0.521 \\
Factor2 & 0.852 & 0.266 & 0.284 & 0.805 \\
Factor3 & 0.586 &. & 0.195 & 1.000 \\
\hline
\end{tabular}

\begin{tabular}{|l|l|l|l|r|}
\hline Factor & Factor1 & Uniqueness & Variable & kmo \\
\hline bildung & 0.571 & 0.674 & bildung & 0.697 \\
lebenserw & 0.790 & 0.377 & lebenserw & 0.557 \\
neunzig_zehn & -0.782 & 0.388 & neunzig_zehn & 0.559 \\
\hline & & & Overall & 0.577 \\
\hline
\end{tabular}

Ergebnisse für das Sub-Sample 2: N=19, FA by principal component factors with varimax. Overall Kaiser-Meyer-Olkin measure of sample adequacy is 0,57 (eigene Berechnung und Darstellung). 
Tabelle A5: Ergebnisse der Hauptkomponentenanalyse am Sub-Sample 3

\begin{tabular}{|l|rrrr|}
\hline Factor & Eigenvalue & Difference & Proportion & Cumulative \\
\hline Factor1 & 1.938 & 1.190 & 0.646 & 0.646 \\
Factor2 & 0.748 & 0.434 & 0.249 & 0.895 \\
Factor3 & 0.314 &. & 0.105 & 1.000 \\
\hline
\end{tabular}

\begin{tabular}{|l|l|l|l|r|}
\hline Factor & Factor1 & Uniqueness & Variable & kmo \\
\hline bip_real_pc & 0.891 & 0.206 & bip_real_pc & 0.559 \\
treibhausg & 0.653 & 0.573 & treibhausg & 0.745 \\
stickstoff & 0.847 & 0.283 & stickstoff & 0.573 \\
\hline & & & Overall & 0.591 \\
\hline
\end{tabular}

Ergebnisse für das Sub-Sample 3: N=20, FA by principal component factors with varimax. Overall Kaiser Meyer-Olkin measure of sample adequacy is 0,59 (eigene Berechnung und Darstellung). 